\title{
Expanded B cell population blocks regulatory T cells and exacerbates ileitis in a murine model of Crohn disease
}

\author{
Timothy S. Olson, ${ }^{1}$ Giorgos Bamias, ${ }^{2}$ Makoto Naganuma, ${ }^{2}$ Jesús Rivera-Nieves, ${ }^{2}$ \\ Tracy L. Burcin, ${ }^{3}$ William Ross, ${ }^{2}$ Margaret A. Morris, ${ }^{3}$ Theresa T. Pizarro, ${ }^{2}$ Peter B. Ernst, ${ }^{2}$ \\ Fabio Cominelli, 2 and Klaus Ley ${ }^{3}$ \\ 1Department of Molecular Physiology and Cardiovascular Research Center, and 2Digestive Health Center of Excellence, University of Virginia \\ Health Science Center, Charlottesville, Virginia, USA. ${ }^{3}$ Department of Biomedical Engineering and Cardiovascular Research Center, \\ University of Virginia, Charlottesville, Virginia, USA.
}

\begin{abstract}
SAMP1/YitFc mice develop discontinuous, transmural inflammatory lesions in the terminal ileum, similar to what is found in human Crohn disease. Compared with the mesenteric lymph nodes (MLNs) of AKR control mice, SAMP1/YitFc MLNs contain a 4.3-fold expansion in total B cell number and a 2.5 -fold increased percentage of $\mathrm{CD}^{+} \mathrm{T}$ cells expressing the $\alpha_{\mathrm{E}} \beta_{7}$ integrin. Although $\alpha_{\mathrm{E}} \beta_{7}{ }^{+} \mathrm{CD} 4^{+} \mathrm{T}$ cells possess a regulatory phenotype $\left(\mathrm{CD}^{+}{ }^{+}, \mathrm{L}_{\text {-selectin }}{ }^{\mathrm{lo}}\right.$, and $\left.\mathrm{CD} 45 \mathrm{RB}^{\mathrm{lo}}\right)$, express IL-10, and suppress effector $\mathrm{T}$ cell proliferation in vitro, they cannot prevent ileitis development in SCID mice adoptively transferred with effector $\mathrm{CD}^{+} \mathrm{T}$ cells, although the $\mathrm{CD4}^{+} \mathrm{CD25}^{+}$subset, which overlaps with the $\alpha_{\mathrm{E}} \beta_{7}{ }^{+} \mathrm{CD} 4^{+}$subset, prevents colitis. The $\alpha_{\mathrm{E}} \beta_{7}{ }^{+} \mathrm{CD} 4^{+} \mathrm{T}$ cells express high levels of ICOS, a costimulatory molecule that augments $B$ cell function, suggesting their involvement in the increase in B cells, IgA ${ }^{+}$cells, and soluble IgA found within the MLNs and ileum of SAMP1/YitFc mice. MLN B cell numbers correlate with ileitis severity in SAMP1/YitFc mice, and cotransfer of SAMP1/YitFc MLN $\mathrm{B}$ cells along with $\mathrm{CD}^{+} \mathrm{T}$ cells increases ileitis severity in SCID mice compared with transfer of $\mathrm{CD}^{+} \mathrm{T}$ cells alone. SAMP1/YitFc B cells prevent $\alpha_{E} \beta_{7}{ }^{+} \mathrm{CD}^{+} \mathrm{T}$ cells from suppressing effector $\mathrm{T}$ cell proliferation. We conclude that SAMP1/YitFc MLN B cells contribute to the development of SAMP1/YitFc ileitis.
\end{abstract}

\section{Introduction}

Inflammatory bowel disease (IBD) requires increased host genetic susceptibility, immune system dysregulation, and altered interactions of host cells with pathogens and normal flora within the intestinal mucosa (1). Although $\mathrm{CD}^{+} \mathrm{T}$ cell subset functions have been studied in great detail (2), the contributions of other immune cells to the development of IBD are just beginning to be understood $(3,4)$. In particular, irregularities in B cell development and antigen-specific immunoglobulin production may be critical for understanding the pathogenesis of IBD (5-7).

Abnormal immunoreactivity of serum or mucosal antibodies toward enteric bacterial flora has been reported in both animal models and IBD patients $(7,8)$. IgA production plays a critical role in preventing the intestinal invasion of both pathogenic and commensal bacteria (9). Although classic follicular B2 lineage cells produce considerable IgA, roughly half of the intestinal IgA-producing plasma cells are derived from B1 cells that develop and differentiate within the peritoneal cavity (10). B1 cells produce IgM or IgA in a T cell-independent manner, with specificities for common enteric bacterial antigens (11). Within lymphoid organs, B2 $\mathrm{B}$ cell proliferation and isotype class switching are promoted by follicular helper $\mathrm{T}$ cells $\left(\mathrm{T}_{\mathrm{FH}}\right.$ cells). Although $\mathrm{T}_{\mathrm{FH}}$ cells lack Th1 or Th2 cytokine production, they greatly enhance B cell IgG and IgA

Nonstandard abbreviations used: follicular helper $\mathrm{T}$ cell ( $\mathrm{T}_{\mathrm{FH}}$ cell); GITR ligand (GITRL); glucocorticoid-induced TNF receptor (GITR); inducible T cell costimulator (ICOS); inflammatory bowel disease (IBD); mesenteric lymph node (MLN); phycoerythrin (PE); regulatory $\mathrm{T}$ cell $\left(\mathrm{T}_{\text {reg }}\right.$ cell)

Conflict of interest: The authors have declared that no conflict of interest exists.

Citation for this article: J. Clin. Invest. 114:389-398 (2004)

doi:10.1172/JCI200420855 production (12). $\mathrm{T}_{\mathrm{FH}}$ cells express high levels of inducible $\mathrm{T}$ cell costimulator (ICOS), a molecule that, through binding of ICOS ligand on B cells, is required for T cell-mediated B cell help and antibody class switching (13).

$\mathrm{CD}^{+} \mathrm{CD} 45 \mathrm{RB}^{\mathrm{lo}} \mathrm{CD} 25^{+}$regulatory $\mathrm{T}$ cells $\left(\mathrm{T}_{\text {reg }}\right.$ cells) prevent colitis induced by transferring effector $\mathrm{CD} 4^{+} \mathrm{CD} 45 \mathrm{RB}^{\text {hi }} \mathrm{CD} 25^{-} \mathrm{T}$ cells into SCID mice through mechanisms involving TGF- $\beta$ and IL-10 (14). Recently, expression of the $\alpha_{E} \beta_{7}$ integrin was used to define novel subsets of $\mathrm{CD}^{+} \mathrm{T}_{\text {reg }}$ cells also capable of preventing colitis (15). The $\alpha_{E} \beta_{7}$ integrin binds E-cadherin on epithelial cells and an unknown ligand on endothelial cells $(16,17)$ and is required for the maintenance of normal lymphocyte numbers within the epithelium and lamina propria (18). In contrast to $\mathrm{CD} 4^{+} \mathrm{CD} 25^{-}$cells, $\mathrm{CD} 4^{+} \mathrm{CD} 25^{+}$ $T_{\text {reg }}$ cells preferentially express $\alpha_{E} \beta_{7}$ (19). These $T_{\text {reg }}$ cells also express high levels of the glucocorticoid-induced TNF receptor (GITR), a TNF receptor family member that regulates $T$ cell proliferation and activation-induced apoptosis (20). Stimulation of $\mathrm{T}_{\text {reg }}$ cells with anti-GITR or through binding of GITR ligand (GITRL), expressed by subsets of APCs, reverses the suppression of effector $\mathrm{T}$ cell proliferation by $\mathrm{T}_{\text {reg }}$ cells in vitro, suggesting a role for GITR in the promotion of proinflammatory responses $(19,21,22)$. Whether this pathway is important in the context of IBD has not been examined.

The SAMP1/YitFc spontaneous ileitis model provides an excellent system for the study of interactions between leukocyte subsets participating in the development of intestinal inflammation. SAMP1/YitFc mice develop Crohn-like discontinuous, transmural ileitis without chemical, genetic, or immunological manipulation (23). The lesions contain many histopathological features seen in Crohn disease, including villous atrophy, crypt hyperplasia, and infiltration of both acute and chronic inflammatory cells (23). 
The lesions are associated with a Th1-type inflammatory response that can be downregulated by antibiotic therapy (24). Importantly, mesenteric lymph node (MLN) $\mathrm{CD}^{+} \mathrm{T}$ cells from SAMP1/YitFc mice adoptively transfer ileitis to SCID recipients (25).

In this study, we have investigated abnormalities in B cell homeostasis and functionality in SAMP1/YitFc mice, beginning with the observation that $\mathrm{B}$ cell numbers are greatly increased in MLNs of SAMP1/YitFc versus wild-type mice. To link B cell population expansion to abnormalities of $T_{\text {reg }}$ cell function in this model, we investigated both $\mathrm{T}$ cell activation of $\mathrm{B}$ cells and the effects of $\mathrm{B}$ cells on $\mathrm{T}$ cell function. To address a causal relationship between B cell population expansion and disease severity, we adoptively transferred $B$ cells along with $T$ cells and measured ileitis. In contrast to models of colitis in which B cells have been shown to decrease disease severity $(5,26)$, our data demonstrate that B cells play an important proinflammatory role in the development of SAMP1/YitFc ileitis through mechanisms that may involve inhibition of $\mathrm{T}_{\text {reg }}$ cell function.

\section{Results}

Expanded B cell and non-naive CD4+ $T$ cell populations in SAMP1/YitFc $M L N s$. One of the hallmarks of the SAMP1/YitFc ileitis phenotype is a macroscopically enlarged MLN (27). SAMP1/YitFc MLNs from mice older than 30 weeks of age had a threefold increase in total lymphocyte number $(52 \pm 5$ million cells, mean \pm SEM) compared with MLNs from age-matched AKR mice (17 \pm 1 million cells) (Figure 1A), the strain from which SAMP1/YitFc mice were derived (23). Using flow cytometry subset analysis, we found the largest expansion occurred in the B cell subset, which was increased 4.3-fold in SAMP1/YitFc compared with AKR MLNs. The $\mathrm{CD}^{+} \mathrm{T}$ cell number was increased 2.5 -fold and, compared with AKR CD4 ${ }^{+} \mathrm{T}$ cells, an increased percentage of SAMP1/YitFc $\mathrm{CD}^{+} \mathrm{T}$ cells expressed CD25 (30\% $\pm 2 \%$ versus $\left.15 \% \pm 1 \%\right)$, and a decreased percentage expressed high levels of L-selectin $(55 \% \pm 4 \%$ versus $84 \% \pm 2 \%)$ and $\mathrm{CD} 45 \mathrm{RB}(55 \% \pm 4 \%$ versus $75 \% \pm 1 \%)$, as described previously (25). Previous studies have shown that $\mathrm{CD} 25^{+} \mathrm{L}$-selectin ${ }^{\mathrm{lo}} \mathrm{CD} 45 \mathrm{RB}^{\mathrm{lo}} \mathrm{CD} 4^{+} \mathrm{T}$ cells can represent either an activated, effector population (28-30) or $\mathrm{T}_{\text {reg }}$ cells $(14,31)$.

$T_{\text {reg }}$ cells can prevent colitis but not ileitis produced by SAMP1/YitFc $\mathrm{CD}^{+} T$ cells. In examining the trafficking capability of SAMP1/ YitFc versus AKR MLN cell subsets, we found an increase in the percentage of SAMP1/YitFc MLN CD4 ${ }^{+} \mathrm{T}$ cells that expressed

\section{Figure 1}

Expanded B cell and $\alpha_{E} \beta_{7}{ }^{+} \mathrm{CD} 4^{+} \mathrm{T}$ cell populations in SAMP1/YitFc versus AKR MLN. (A) Total lymphocyte numbers (mean \pm SEM) presented as the percentage of total cells in AKR and SAMP1/YitFC MLNs - as determined by lymphocyte-gated flow cytometry - that are CD4+ T cells ( $n=16$ and 32, respectively), CD ${ }^{+}$T cells $(n=10$ and 19 , respectively), and B cells ( $n=13$ and 24 , respectively). Fold increases in the overall size of each of these populations in SAMP1/ YitFc versus AKR are indicated. (B) Top, CD4 ${ }^{+} \mathrm{T}$ cell-gated histograms of $\beta_{7}$ integrin chain expression on MLN cells from SAMP1/YitFc and AKR mice, showing that SAMP1/YitFc mice have an increased percentage of $\mathrm{CD} 4^{+} \mathrm{T}$ cells expressing high levels of $\beta_{7}$. Bottom, the $\beta_{7}{ }^{\text {hi }}$ cells express $\beta_{7}$ as a dimer with the $\alpha_{E}$ integrin chain, as $\alpha_{E}{ }^{+}$cells display a 1:1 correlation of $\alpha_{E}$ to $\beta_{7}$ expression in $\beta_{7}$ versus $\alpha_{E}$ dot plots that is not seen in isotype controls. (C) Comparison of the percentage (mean \pm SEM) of MLN CD4 ${ }^{+}$T cells that are $\alpha_{E^{+}}$in SAMP1/YitFc mice $(n=31)$ versus AKR mice $(n=21)$. *Significantly greater $(P<0.05)$ than AKR cell percentage. high levels of the $\beta_{7}$ integrin subunit (Figure $2 \mathrm{~A}$, histograms), which can form $\alpha_{4} \beta_{7}$, an integrin required for trafficking of lymphocytes to intestinal tissues (32), or $\alpha_{\mathrm{E}} \beta_{7}$. Although virtually all $\mathrm{CD}^{+} \mathrm{T}$ cells from SAMP1/YitFc or AKR MLNs expressed low levels of $\alpha_{4} \beta_{7}$ when detected at sufficient intensity by flow cytometry (data not shown), only a subset of SAMP1/YitFc cells expressed $\alpha_{E}$, and this expression correlated directly with high levels of $\beta_{7}$ expression (Figure $1 \mathrm{~B}$, dot plots), showing that $\beta_{7}^{\text {hi }}$ cells specifically express the $\alpha_{E} \beta_{7}$ dimer. The percentage of MLN $\mathrm{CD}^{+} \mathrm{T}$ cells expressing $\alpha_{\mathrm{E}} \beta_{7}$ was increased 2.5 -fold in SAMP1/ YitFc mice $(15 \% \pm 1 \%$, mean \pm SEM) compared with AKR mice $(6.0 \% \pm 0.5 \%)$ (Figure $2 \mathrm{~B})$. Although the majority of $\alpha_{\mathrm{E}} \beta_{7}{ }^{-} \mathrm{CD} 4^{+}$ cells were CD69- $(82 \% \pm 2 \%)$, CD25- $^{-}(79 \pm 3 \%)$, L-selectin $^{\text {hi }}$ $(58 \% \pm 5 \%)$, and CD $45 \mathrm{RB}^{\text {hi }}(53 \% \pm 6 \%)$, most $\alpha_{\mathrm{E}} \beta_{7^{+}} \mathrm{CD}^{+}$cells were $\mathrm{CD} 9^{+}(59 \% \pm 3 \%), \mathrm{CD} 25^{+}(75 \% \pm 3 \%)$, L-selectin $^{\mathrm{lo}}(66 \% \pm 4 \%)$, and $\mathrm{CD}_{45 \mathrm{RB}^{\mathrm{lo}}}(74 \% \pm 6 \%)$ (Figure $\left.2 \mathrm{~A}\right)$, consistent with a regulatory phenotype (15). To test whether this expanded $\alpha_{\mathrm{E}} \beta_{7}{ }^{+} \mathrm{CD} 4^{+} \mathrm{T}$ cell subset possessed regulatory or ileitis-producing capability, we analyzed the cytokine expression of isolated MLN $\alpha_{E} \beta_{7}{ }^{+} \mathrm{CD} 4^{+}$ versus $\alpha_{\mathrm{E}} \beta_{7}{ }^{-} \mathrm{CD} 4^{+} \mathrm{T}$ cells in 2 -day cultures by cytometric bead

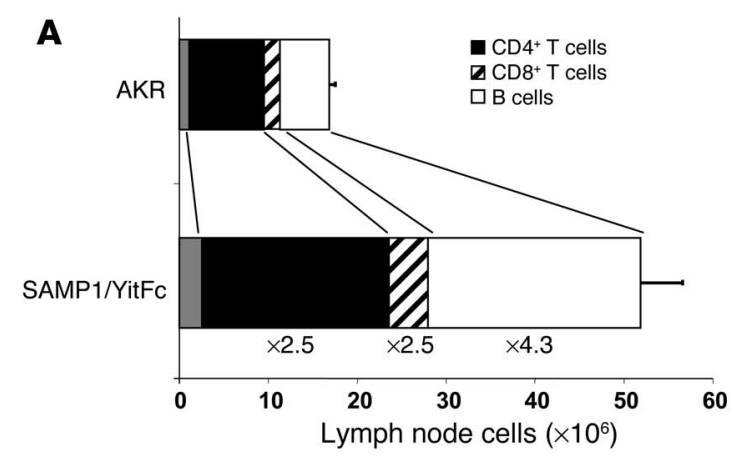

$\mathbf{B}$
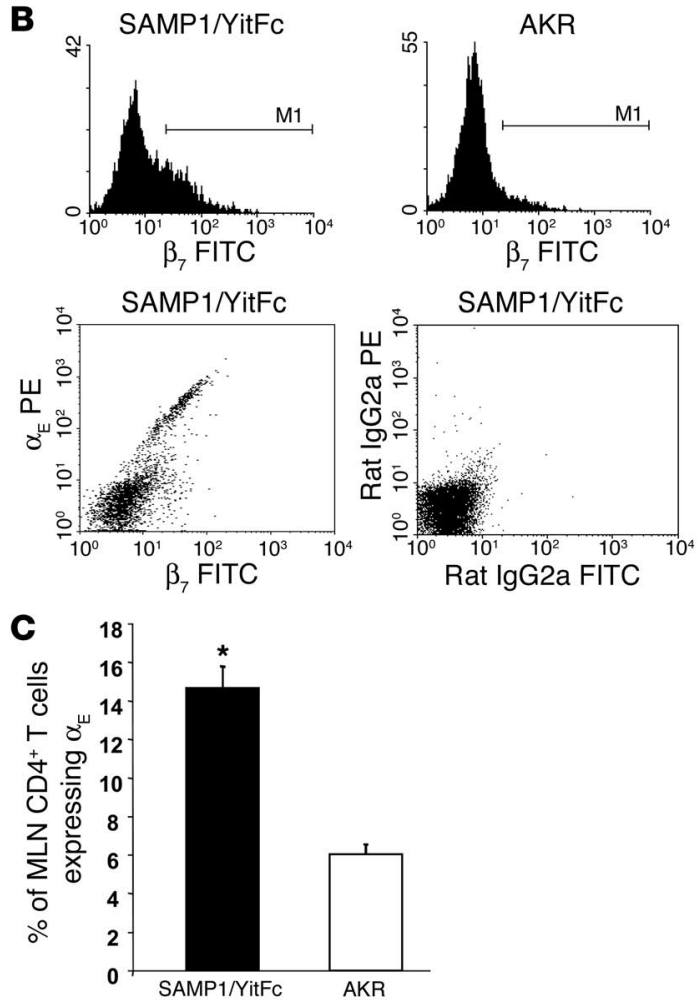

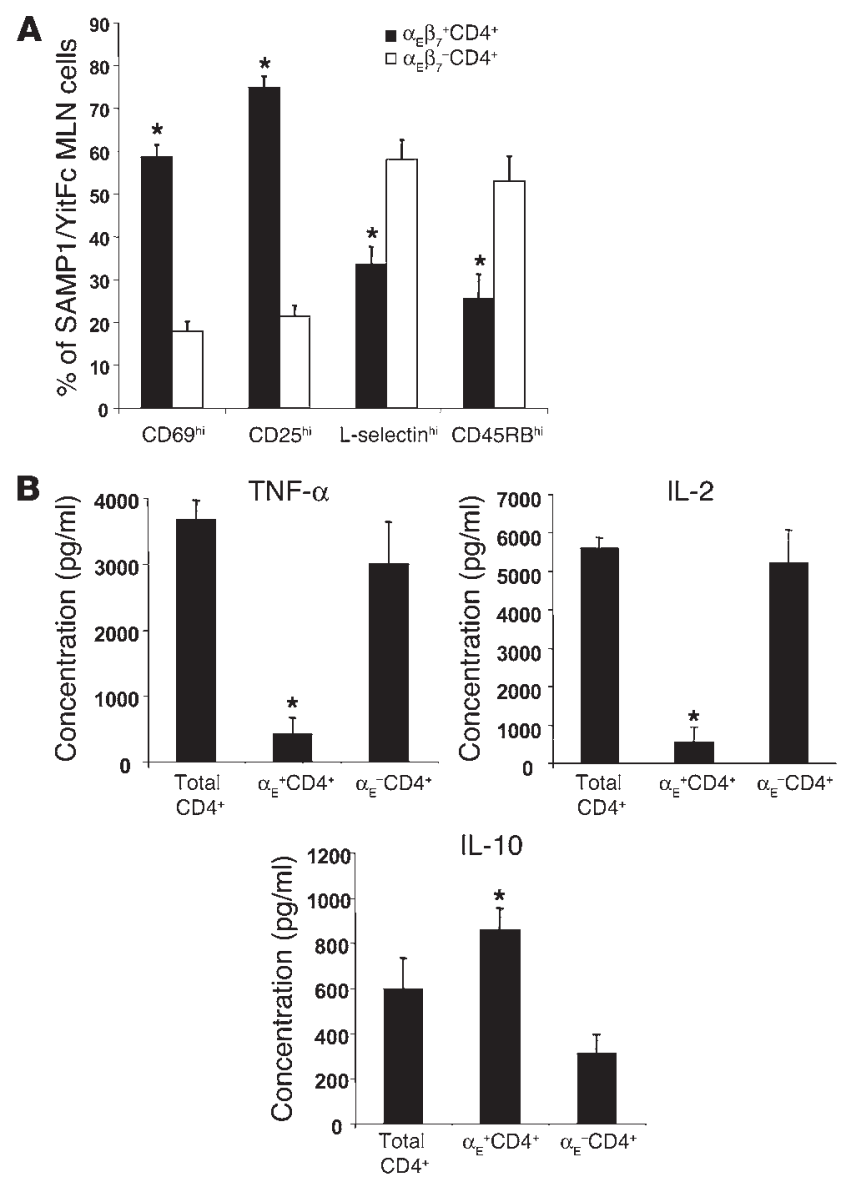

Figure 2

SAMP1/YitFc MLN $\alpha_{E} \beta_{7}+C D 4+T$ cells possess a regulatory phenotype. (A) Comparison of the percentage of MLN cells, determined by flow cytometry, that express high levels of CD69 ( $n=9$ mice), CD25 $(n=9)$, L-selectin $(n=14)$, and CD45RB $(n=8)$ within the $\alpha_{E} \beta_{7}+C D 4^{+}$and $\alpha_{E} \beta 7^{-}$ CD4 ${ }^{+}$T cell subsets. (B) Levels of secreted TNF- $\alpha$, IL-2, and IL-10, measured by cytometric bead array in triplicate, from 48 -hour cultures of SAMP1/YitFc MLN unfractionated CD4+ ${ }^{+}$cells (Total CD4+), $\alpha_{E}{ }^{+C D} 4{ }^{+} T$ cells, or $\alpha_{E}{ }^{-C D} 4+T$ cells $\left(10^{5}\right.$ cells/well), stimulated with immobilized anti-CD3 and soluble anti-CD28. Values represent the averages of three independent experiments for each group. *Significantly different $(P<0.05)$ compared to $\alpha_{E} \beta_{7}{ }^{-} \mathrm{CD} 4^{+} T$ cell population.

array. The $\alpha_{\mathrm{E}} \beta_{7}{ }^{+} \mathrm{CD} 4^{+} \mathrm{T}$ cells produced eight- to tenfold lower levels of TNF- $\alpha$ and IL-2 and almost threefold greater amounts of IL-10 than did $\alpha_{E} \beta_{7}{ }^{-} \mathrm{CD} 4^{+} \mathrm{T}$ cells (Figure $2 \mathrm{~B}$ ).

To test the function of SAMP1/YitFc MLN $\alpha_{\mathrm{E}} \beta_{7}{ }^{+} \mathrm{CD} 4^{+} \mathrm{T}$ cells, we adoptively transferred $5 \times 10^{5}$ unfractionated, $\alpha_{\mathrm{E}} \beta_{7}{ }^{+} \mathrm{CD} 4^{+}$, or $\alpha_{\mathrm{E}} \beta_{7}$ $\mathrm{CD}^{+}{ }^{+} \mathrm{T}$ cells intraperitoneally into $\mathrm{C} 3 \mathrm{H} / \mathrm{HeJ}$ SCID mice. After 6 weeks, ilea were removed from the recipients and analyzed for ileitis severity with a standardized histopathological scoring system (33) (Figure 3A). Compared with mice receiving SAMP1/YitFc $\alpha_{\mathrm{E}} \beta_{7}{ }^{-} \mathrm{CD} 4^{+} \mathrm{T}$ cells, SCID mice receiving SAMP1/YitFc $\alpha_{\mathrm{E}} \beta_{7}{ }^{+} \mathrm{CD} 4^{+}$ $\mathrm{T}$ cells had significantly lower chronic inflammatory indices $(1.3 \pm 0.3$ versus $5.6 \pm 0.4$, mean \pm SEM $)$ and total inflammatory scores ( $8 \pm 1$ versus $21 \pm 1$ ), suggesting that $\alpha_{E} \beta_{7}{ }^{-} C D 4^{+} T$ cells contain the major ileitis-producing subset.

Since the percentage of CD $4^{+} \mathrm{T}$ cells expressing $\alpha_{\mathrm{E}} \beta_{7}$ increases with age in SAMP1/YitFc MLNs (data not shown) and the severity of SAMP1/YitFc ileitis stabilizes with age (27), $\alpha_{\mathrm{E}} \beta_{7^{+}} \mathrm{CD} 4^{+} \mathrm{T}$ cell populations might expand in an attempt to curb inflammation in older SAMP1/YitFc mice. To test whether $\alpha_{\mathrm{E}} \beta_{7}{ }^{+} \mathrm{CD} 4^{+} \mathrm{T}$ cells can downregulate ileitis, we injected $5 \times 10^{5} \alpha_{\mathrm{E}} \beta_{7}{ }^{+} \mathrm{CD} 4^{+} \mathrm{T}$ cells into SCID mice 3 weeks before, at the same time as, or 3 weeks after injection of $5 \times 10^{5} \alpha_{\mathrm{E}} \beta_{7^{-}} \mathrm{CD} 4^{+} \mathrm{T}$ cells (Figure $3 \mathrm{~A}$ ). No changes were seen in the inflammatory scores in any of the three groups compared with scores obtained from mice receiving $\alpha_{\mathrm{E}} \beta_{7}{ }_{7} \mathrm{CD} 4^{+} \mathrm{T}$ cells alone, indicating that SAMP1/YitFc $\alpha_{\mathrm{E}} \beta_{7}{ }^{+} \mathrm{CD} 4^{+} \mathrm{T}$ cells cannot downregulate ileitis produced by $\alpha_{E} \beta_{7}{ }^{-} C D 4^{+}$T cells. To test whether SAMP1/YitFc $\alpha_{\mathrm{E}} \beta_{7^{+}} \mathrm{CD} 4^{+} \mathrm{T}$ cells might have defective regulatory function compared with wild-type regulatory cells, we transferred $5 \times 10^{5}$ SAMP1/ YitFc CD $4^{+} \mathrm{T}$ cells alone or a combination of SAMP1/YitFc plus AKR $\left(5 \times 10^{5}\right.$ cells each $) \mathrm{CD}^{+} \mathrm{T}$ cells into a separate cohort of SCID mice (Figure $3 \mathrm{~B}$ ). AKR MLN CD4 ${ }^{+} \mathrm{T}$ cells were unable to prevent the ileitis produced by SAMP1/YitFc CD4 ${ }^{+} \mathrm{T}$ cells.

To test regulatory cell function in the well characterized CD45RB ${ }^{\text {hi }} \mathrm{CD}^{+}$and $\mathrm{CD} 25^{+} \mathrm{CD} 4^{+} \mathrm{T}$ cell adoptive transfer models (14) using $T_{\text {reg }}$ populations that overlap with the $\alpha_{E}{ }^{+} C D 4^{+}$population (15), we transferred $5 \times 10^{5}$ unfractionated, CD45RB hi, CD45RB ${ }^{\text {lo }}, \mathrm{CD}_{25}{ }^{+}$, or CD25- SAMP1/YitFc MLN CD4 ${ }^{+} \mathrm{T}$ cells into a third cohort of SCID mice and determined both ileitis and colitis severity (Figure $3 \mathrm{C}$ ). $\mathrm{CD} 45 \mathrm{RB}^{\mathrm{hi}} \mathrm{CD}^{+}$or $\mathrm{CD} 25^{-} \mathrm{CD} 4^{+} \mathrm{T}$ cells produced levels of ileitis similar to that produced by unfractionated $\mathrm{CD}^{+}$cells. $\mathrm{CD} 25^{+} \mathrm{CD} 4^{+} \mathrm{T}$ cells produced significantly less ileitis than unfractionated $\mathrm{CD}^{+} \mathrm{T}$ cells. $\mathrm{CD} 45 \mathrm{RB}^{\mathrm{lo}} \mathrm{CD} 4^{+} \mathrm{T}$ cells produced levels of ileitis similar to that produced by $\mathrm{CD} 45 \mathrm{RB}^{\text {hi }} \mathrm{CD} 4^{+}$ $\mathrm{T}$ cells, suggesting that $\mathrm{CD} 45 \mathrm{RB}^{\text {lo }} \mathrm{CD} 4^{+} \mathrm{T}$ cells contain subsets of cells that are proinflammatory in the context of ileitis.

In contrast, SCID mice receiving SAMP1/YitFc MLN $\mathrm{CD} 45 \mathrm{RB}^{\mathrm{hi}} \mathrm{CD} 4^{+}$or $\mathrm{CD} 25^{-} \mathrm{CD} 4^{+} \mathrm{T}$ cells exhibited substantially more colitis than mice receiving unfractionated $\mathrm{CD}^{+} \mathrm{T}$ cells containing $\mathrm{T}_{\text {reg }}$ cells (Figure 3C). This finding strongly suggests that SAMP1/ YitFc CD4 $4^{+} \mathrm{T}_{\text {reg }}$ cell populations (CD45RB low, $\mathrm{CD} 25^{+}$, or $\alpha_{\mathrm{E}}^{+}$) are not defective, as they can suppress the development of colitis in a fashion similar to $\mathrm{T}_{\text {reg }}$ cells in other adoptive transfer models.

$\alpha_{E} \beta 7_{7}^{+} C D 4^{+} T$ cells may be involved in $B$ cell help. We next tested whether $\alpha_{\mathrm{E}} \beta_{7}{ }^{+} \mathrm{CD} 4^{+} \mathrm{T}$ cells might be involved in the B cell population expansion seen in SAMP1/YitFc MLNs. In contrast to the majority of $\alpha_{\mathrm{E}} \beta_{7}{ }^{-} \mathrm{CD} 4^{+} \mathrm{T}$ cells, most SAMP1/YitFc $\alpha_{\mathrm{E}} \beta_{7^{+}} \mathrm{CD} 4^{+} \mathrm{T}$ cells $(73 \% \pm 2 \%$, mean \pm SEM) expressed ICOS, a costimulatory molecule that plays a critical role in germinal center formation and immunoglobulin class switching (34) (Figure 4B). We found a positive correlation between the percentage of MLN CD4 ${ }^{+} \mathrm{T}$ cells that expressed $\alpha_{\mathrm{E}}$ and MLN B cell number, as a percentage of total cells, in individual mice (Figure 4A). In mice in which more than $15 \%$ of $\mathrm{CD}^{+} \mathrm{T}$ cells expressed $\alpha_{\mathrm{E}} \beta_{7}$, B cells comprised $55 \% \pm 4 \%$ (mean \pm SEM) of total MLN lymphocytes, whereas in mice in which $\alpha_{\mathrm{E}}^{+}$cells constituted less than $15 \%$ of the CD4 $4^{+}$population, only $34 \% \pm 3 \%$ of total MLN lymphocytes were B cells. This correlation, along with the high level of ICOS expression by $\alpha_{\mathrm{E}} \beta_{7}{ }^{+} \mathrm{CD} 4^{+} \mathrm{T}$ cells, suggests that this subset may directly interact with $\mathrm{B}$ cells in SAMP1/YitFc MLNs.

SAMP1/YitFc B cells produce increased IgA. Compared with AKR supernatants, SAMP1/YitFc MLN supernatants contained more $\operatorname{IgG}_{1} \kappa$ (3.4-fold), $\operatorname{IgA} \kappa$ (4.5-fold), $\operatorname{IgM} \kappa$ (2.3-fold), $\operatorname{IgA} \gamma$ (7.9-fold), and $\operatorname{IgM} \gamma$ (3.7-fold), as determined by cytometric bead array (Figure $5 \mathrm{~A}$ ). Cocultures of SAMP1/YitFc CD4 ${ }^{+} \mathrm{T}$ cells and B cells contained more IgA and IgM, but not IgG2a, than did cocultures of AKR CD4 ${ }^{+} \mathrm{T}$ cells and B cells (Figure $5 \mathrm{C}$ ), recapitulating the in vivo expression pattern. Mixed cocultures of SAMP1/YitFc CD4 ${ }^{+}$ 


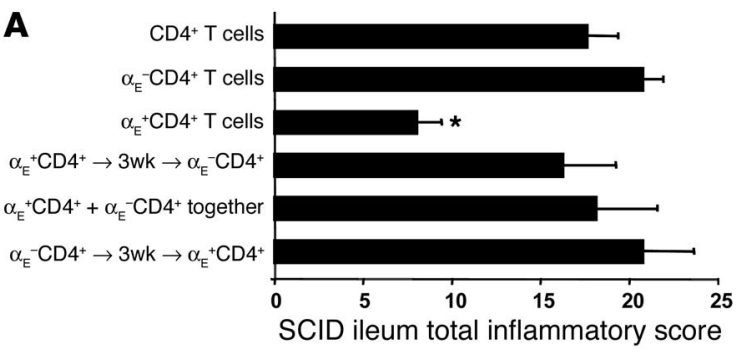

B
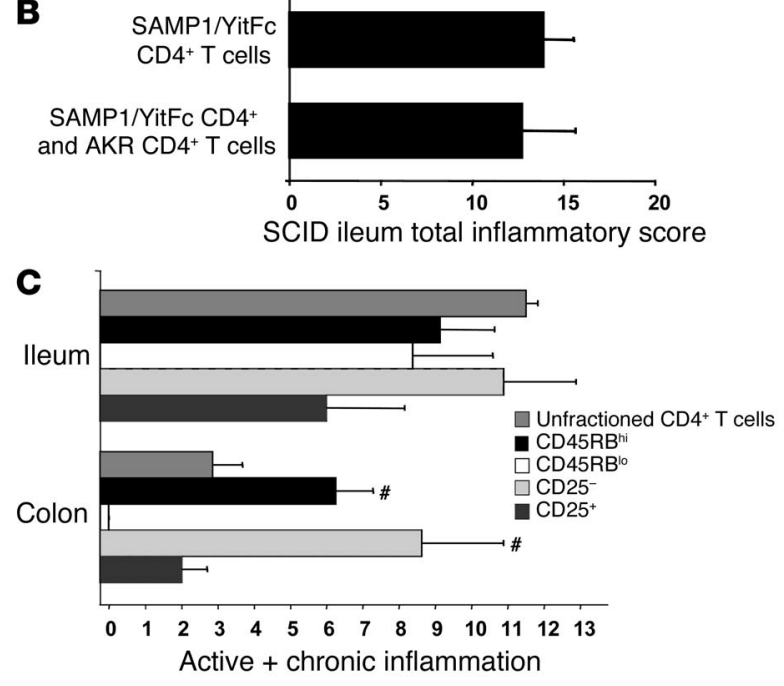

$\mathrm{T}$ cells plus AKR B cells, as well as those of AKR $\mathrm{CD} 4^{+} \mathrm{T}$ cells plus SAMP1/YitFc B cells, produced levels of IgA similar to that of cocultures of AKR T cells and AKR B cells, suggesting that both SAMP1/YitFc B cells and T cells are required for the SAMP1/YitFc expression pattern (data not shown). SAMP1/YitFc B cells cultured with $\alpha_{\mathrm{E}}{ }^{+} \mathrm{CD} 4^{+} \mathrm{T}$ cells produced mostly IgA, whereas B cells cultured with $\alpha_{\mathrm{E}}{ }^{-} \mathrm{CD} 4^{+} \mathrm{T}$ cells produced increased IgM and IgG2a (data not shown). Serum IgA was elevated 2.7-fold in SAMP1/ YitFc mice $(23 \pm 5 \mu \mathrm{g} / \mathrm{ml}$, mean \pm SEM $)$ compared with AKR mice $(8.7 \pm 0.8 \mu \mathrm{g} / \mathrm{ml})$ (Figure $5 \mathrm{~B})$, suggesting that the increase in MLN IgA production may lead to elevated systemic levels.

Immunostaining and flow cytometry showed that SAMP1/YitFc MLNs contained many more IgA ${ }^{+}$cells than did AKR MLNs (Figure $6, A$ and $B$ ). Most of the IgA expression was found on mature $B$ cells (B220 hi ), while some was also found on B220 int cells thought to be differentiating plasmablasts (35). $\operatorname{IgA}^{+}$cells were $\operatorname{IgM}^{+}$and Syndecan- $1^{-}$(data not shown), suggesting that these cells had yet to differentiate into plasma cells. Less than $20 \%$ of SAMP1/YitFc MLN B cells possessed a B1 cell phenotype (CD23- $\left.\mathrm{IgD}^{-}\right)$, indicating that most MLN B cells belonged to the B2 class. The CD23-IgD-

\section{Figure 4}

Correlation between B cell expansion and $\alpha_{E} \beta_{7}{ }^{+} C D 4^{+} T$ cells. (A) Left, correlation $(r=0.6)$ between B cell number as a percentage of all MLN cells and the percentage of MLN CD4 $4^{+}$cells expressing $\alpha_{E}$ in individual SAMP1/YitFc mice $(n=21)$. Right, comparison of the percentage of $B$ cells in MLNs of mice with $\alpha_{E}{ }^{+} C D 4^{+}$cells comprising greater than versus less than $15 \%$ of the MLN CD4+ population (line represents mean). (B) Representative CD4+-gated dot plot and average quadrant percentages $(n=10)$ showing expression of $\beta_{7}$ integrin versus ICOS on SAMP1/YitFc MLN CD4+ ${ }^{+}$cells.

\section{Figure 3}

SAMP1/YitFc $T_{\text {reg }}$ populations block colitis, but not ileitis, in the CD4 ${ }^{+} \mathrm{T}$ cell adoptive transfer model. (A) Comparison of adoptively transferred ileitis severity ( 6 weeks after transfer, mean \pm SEM) in SCID recipients ( $n=4$ in each group) induced by $5 \times 10^{5}$ SAMP1/YitFc total MLN CD4 ${ }^{+}$ $T$ cells, $\alpha_{E}{ }^{-C D} 4^{+} T$ cells, $\alpha_{E}{ }^{+} C D 4^{+} T$ cells, or combination treatment using $\alpha_{E}{ }^{+} C D 4^{+} T$ cells injected 3 weeks (3wk) before, at the same time as, or 3 weeks after $\alpha_{E}{ }^{-C D} 4^{+} T$ cells. (B) In a separate cohort of SCID mice, severity of ileitis induced by $5 \times 10^{5}$ SAMP1/YitFc CD4 ${ }^{+} \mathrm{T}$ cells $(n=4)$ was not decreased by coinjection of $5 \times 10^{5}$ AKR MLN CD4+ T cells $(n=5)$. Total inflammatory scores represent the sum of three individual histological indices, including active inflammation, chronic inflammation, and villus architectural distortion. (C) In a third cohort, adoptively transferred ileitis and colitis severities were compared among SCID mice 6 weeks after mice received $5 \times 10^{5}$ SAMP1/YitFC

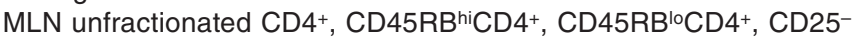
$\mathrm{CD}^{+}$, or $\mathrm{CD} 25^{+} \mathrm{CD} 4^{+} \mathrm{T}$ cells. Because villus distortion is not measured in colitis, the sum of active and chronic inflammatory scores was used for this comparison. Data are expressed as mean \pm SEM. *Significantly decreased $(P<0.05)$ compared with ileitis severity in mice receiving $\alpha_{E} \beta_{7}-C D 4+$ cells. \#Significantly increased compared with colitis severity in mice receiving unfractionated $\mathrm{CD} 4{ }^{+} \mathrm{T}$ cells.

population may also include memory or early antigen-secreting cells derived from $\mathrm{B} 2$ cells, suggesting that the $\mathrm{B} 1$ population may be even smaller than the $\mathrm{CD}^{2} 3^{+} \mathrm{IgD}{ }^{-}$populations seen in Figure $6 \mathrm{~B}$. Most $\mathrm{IgA} \mathrm{A}^{+}$cells were found in the $\mathrm{CD} 23^{+} \mathrm{B} 2$ cell population (data not shown). In contrast to the $\mathrm{B}$ cell and $\mathrm{IgA}^{+}$cell population expansion in SAMP1/YitFc versus AKR MLNs, spleens and Peyer's patches of SAMP1/YitFc versus AKR mice contained similar percentages of $\mathrm{B}$ cells as well as IgA $\mathrm{A}^{+}$cells (data not shown).

In AKR ileal sections, IgA ${ }^{+}$cells were found as single cells or in small clusters, evenly distributed throughout the intestine (Figure 6, C and D). Most of the free IgA staining was located within crypts or near the base of villi. In SAMP1/YitFc ilea, IgA ${ }^{+}$cells were found in large clusters and were distributed in a focally concentrated pat-
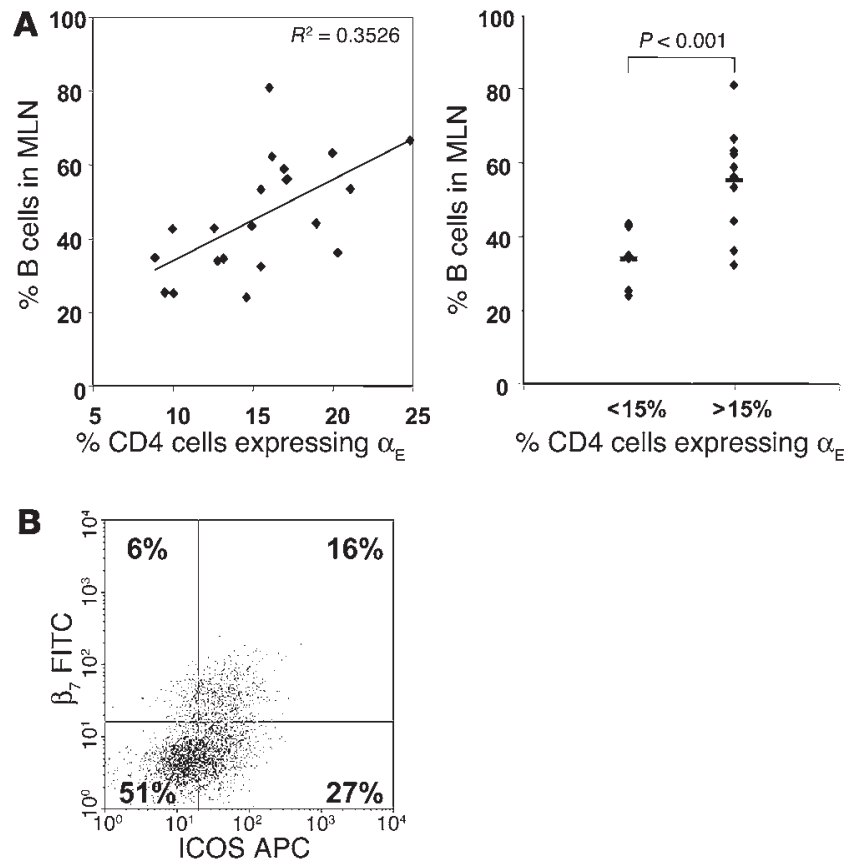
A
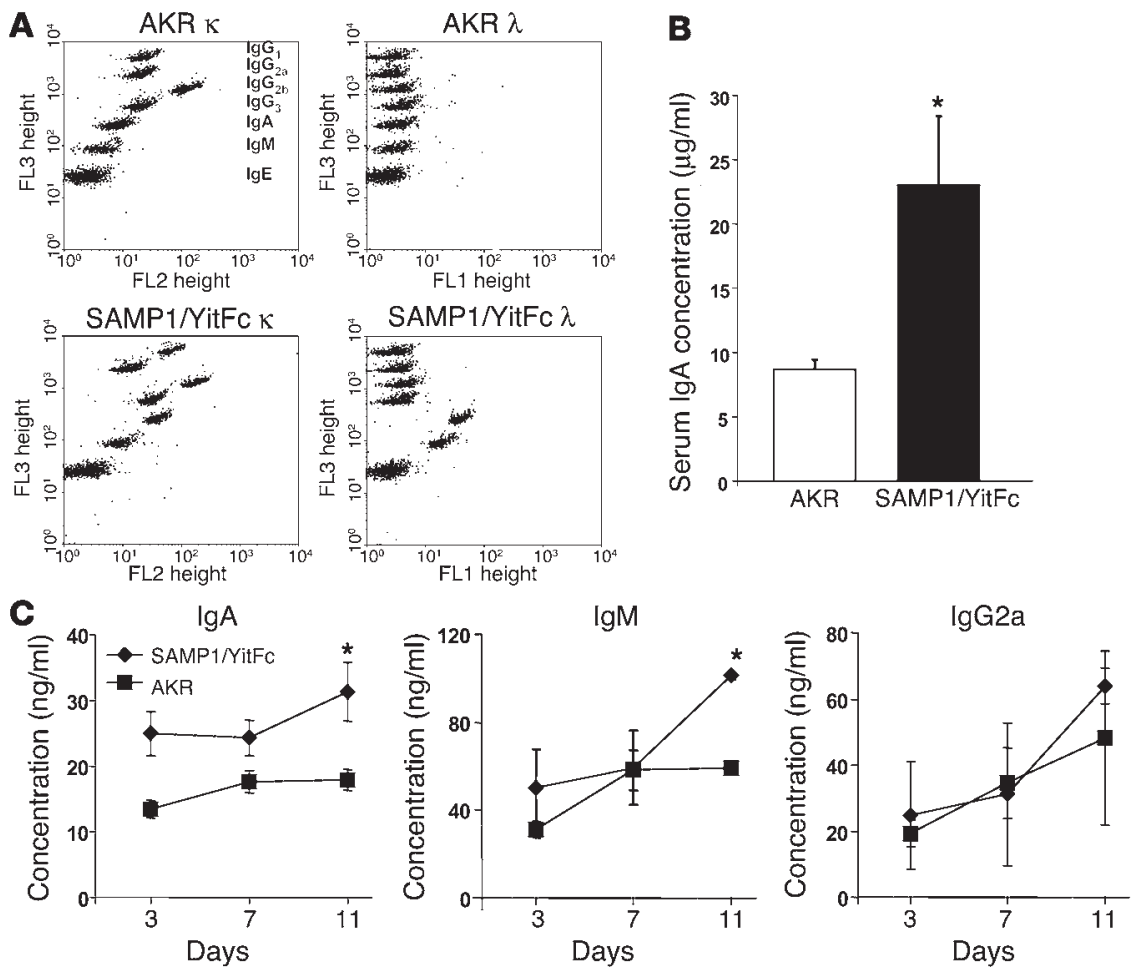

Figure 5

Soluble IgA production increased in SAMP1/YitFc versus AKR mice. (A) Representative dot plots of $\kappa(1: 100$ dilution, FL2) and $\lambda(1: 1, F L 1)$ light chain antibody isotype levels within MLN supernatants from AKR mice $(n=2)$ and SAMP1/YitFc mice $(n=2)$, measured with a cytometric bead array containing isotype-specific beads with preset FL3 intensities. MLNs were crushed, resuspended in $5 \mathrm{ml}$, and centrifuged to remove the cell pellet from the tested supernatants. An increase of at least twofold in $\lg G 1 \kappa, \lg A \kappa, \lg M \kappa, \lg A \lambda$, and $\lg M \lambda$ was seen in SAMP1/YitFc versus AKR MLNs, as measured by mean fluorescence intensity of the isotype-specific beads. (B) ELISA detecting IgA antibody concentrations (mean \pm SEM) in serum samples collected via cardiac puncture from SAMP1/YitFc mice $(n=12)$ and wild-type AKR mice $(n=13)$. (C) Concentrations of $\operatorname{IgA}(n=4)$, IgM $(n=2)$, and IgG2a $(n=2)$, measured in triplicate by ELISA, from 3-, 7-, and 11-day anti-CD3-stimulated cocultures of SAMP1/YitFc CD4+ T cells and B cells versus AKR CD4 ${ }^{+} \mathrm{T}$ cells and $\mathrm{B}$ cells $\left(10^{5} \mathrm{~T}\right.$ cells/well and $10^{5} \mathrm{~B}$ cells/well). ${ }^{*}$ Significantly greater than AKR concentrations $(P<0.05)$.

tern. Most of these clusters of $\operatorname{Ig} \mathrm{A}^{+}$cells were found in areas where the tissue architecture was only moderately distorted.

Contribution of B cells to SAMP1/YitFc and adoptively transferred ileitis. To test whether MLN B cells contribute to SAMP1/YitFc ileitis, we first compared $B$ cell population expansion in the MLNs to ileitis severity in the same mouse (Figure 7A). In SAMP1/YitFc mice that exhibited mild ileal inflammation (total ileal inflammatory scores $<10$ ), there was a ratio of almost 2:1 of $\mathrm{CD}^{+} \mathrm{T}$ cells $(54 \% \pm 1 \%$, mean \pm SEM) to B cells $(30 \% \pm 1 \%)$ as a percentage of total MLN lymphocytes. In SAMP1/YitFc mice that exhibited severe ileal inflammation (total scores $>10$ ), the $\mathrm{B}$ cell population $(42 \% \pm 4 \%)$ exceeded the number of $\mathrm{CD}^{+} \mathrm{T}$ cells $(38 \% \pm 3 \%)$ within the MLNs. Multiple regression analysis revealed that B cell population expansion within the MLNs correlated most closely with the villus distortion index (Figure 7B). To test whether SAMP1/YitFc MLN B cells were pro- or anti-inflammatory, we cotransferred $5 \times 10^{5}$ SAMP1/YitFc MLN CD4 ${ }^{+} \mathrm{T}$ cells with or without $2 \times 10^{6} \mathrm{SAMP} 1 / \mathrm{YitFc}$ or AKR MLN B cells into SCID recipients (Figure $8 \mathrm{~A}$ ). Mice receiving $\mathrm{CD} 4^{+} \mathrm{T}$ cells and SAMP1/YitFc B cells had significantly greater chronic inflamma- tory indices and total inflammatory scores than those of mice receiving $\mathrm{T}$ cells alone. SCID mice receiving AKR B cells along with SAMP1/YitFc CD4 ${ }^{+} \mathrm{T}$ cells displayed an "intermediate" phenotype, with similar levels of chronic inflammation but significantly decreased active inflammation compared with mice receiving SAMP1/YitFc B cells and $\mathrm{CD}^{+} \mathrm{T}$ cells. Only scattered $\mathrm{IgA}^{+}$ cells were found in ileal sections of mice receiving both SAMP1/YitFc T cells and $\mathrm{B}$ cells, although around $10 \%$ of MLN cells in these mice expressed IgA (data not shown). Immunostaining of ileal sections revealed a large increase in $\mathrm{CD}^{+}$cellular infiltrate size in mice receiving SAMP1/YitFc CD4 ${ }^{+}$ $\mathrm{T}$ cells along with either SAMP1/YitFc or AKR B cells compared with that of mice receiving $\mathrm{T}$ cells alone (Figure $\mathrm{BB}$ ). In contrast, a heavy submucosal neutrophil infiltrate was observed only in mice receiving SAMP1/YitFc, but not AKR, B cells.

SAMP1/YitFc MLN B cells suppress $T_{\text {reg }}$ cell function. The increase in $\mathrm{CD}^{+}$infiltrates in SCID mice receiving both $\mathrm{CD} 4^{+} \mathrm{T}$ cells and $B$ cells suggests that B cells may promote effector $\mathrm{T}$ cell proliferation. Because interactions with GITRL can inhibit GITR $^{+} \mathrm{T}_{\text {reg }}$ cell suppression of effector cell proliferation (21), we examined the expression of GITR on SAMP1/YitFc $\alpha_{\mathrm{E}}{ }^{+} \mathrm{CD} 4^{+} \mathrm{T}$ cells by flow cytometry and GITRL on MLN B cells by real-time RT-PCR. The majority of freshly isolated $\alpha_{\mathrm{E}}{ }^{+} \mathrm{CD} 4^{+} \mathrm{T}$ cells expressed GITR, while most $\alpha_{\mathrm{E}}{ }^{-} \mathrm{CD} 4^{+} \mathrm{T}$ cells did not (Figure 9A). The $\alpha_{\mathrm{E}}^{+} \mathrm{CD} 4^{+} \mathrm{T}$ cells from anti-CD3stimulated cultures also expressed higher levels of GITR than did the remaining $\mathrm{CD}^{+}$ population. SAMP1/YitFc MLN B cells expressed mRNA encoding GITRL at higher levels than AKR MLN B cells (Figure 9B).

We next tested whether B cells could interfere with $\alpha_{\mathrm{E}}{ }^{+} \mathrm{CD} 4^{+} \mathrm{T}$ cell regulatory function by measuring cell proliferation in 3-day cultures of irradiated APCs and SAMP1/YitFc cell subsets (Figure 9C). Because $\mathrm{B}$ cells alone did not proliferate and the addition of B cells to either $\alpha_{\mathrm{E}}{ }^{+} \mathrm{CD} 4^{+}$or $\alpha_{\mathrm{E}}{ }^{-} \mathrm{CD} 4^{+} \mathrm{T}$ cells alone did not significantly increase the proliferation in these wells, it is unlikely that $B$ cells underwent proliferation in this assay. The $\alpha_{\mathrm{E}}^{-} \mathrm{CD} 4^{+} \mathrm{T}$ cells showed strong proliferation when cultured with APCs alone, and the addition of $\alpha_{\mathrm{E}}^{+} \mathrm{CD} 4^{+} \mathrm{T}$ cells markedly suppressed this proliferation, confirming that SAMP1/YitFc $\alpha_{\mathrm{E}}^{+} \mathrm{CD} 4^{+} \mathrm{T}$ cells are functional regulatory cells. The addition of $\mathrm{B}$ cells to cultures containing both $\alpha_{\mathrm{E}}{ }^{+} \mathrm{CD} 4^{+}$and $\alpha_{\mathrm{E}}{ }^{-} \mathrm{CD} 4^{+} \mathrm{T}$ cells reversed the suppressive effect of $\alpha_{\mathrm{E}}{ }^{+} \mathrm{CD} 4^{+} \mathrm{T}$ cells, leading to levels of ${ }^{3} \mathrm{H}$ incorporation similar to that of cultures of effector $\alpha_{\mathrm{E}}{ }^{-} \mathrm{CD} 4^{+} \mathrm{T}$ cells alone.

\section{Discussion}

In SAMP1/YitFc mice, an expanded $\alpha_{\mathrm{E}} \beta_{7}{ }^{+} \mathrm{CD} 25^{+} \mathrm{CD} 45 \mathrm{RB}^{\text {lo }} \mathrm{CD} 4^{+}$ MLN T cell subset is associated with increased $B$ cells that contribute to ileitis. These $\mathrm{T}$ cells have a phenotype similar to that 
A SAMP1/YitFc MLN IgA
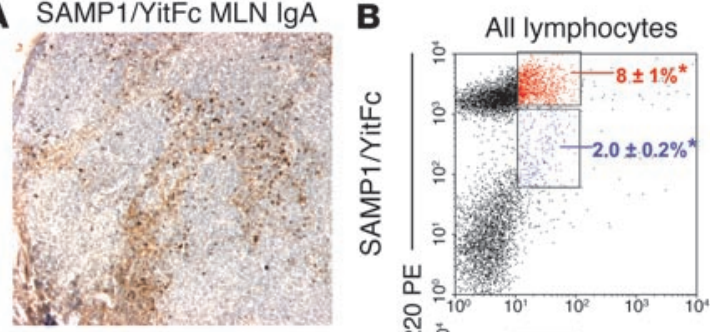

AKR MLN IgA
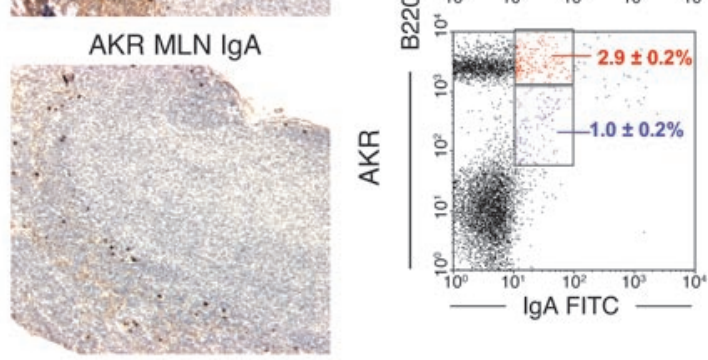

ลิ
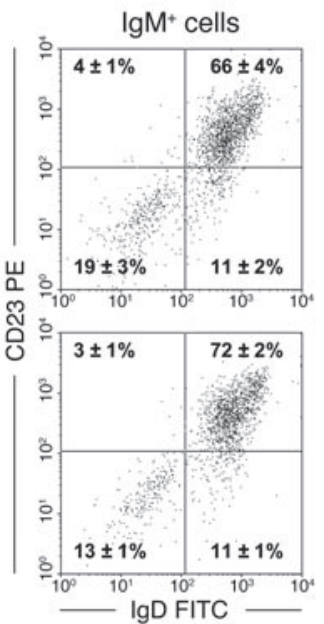
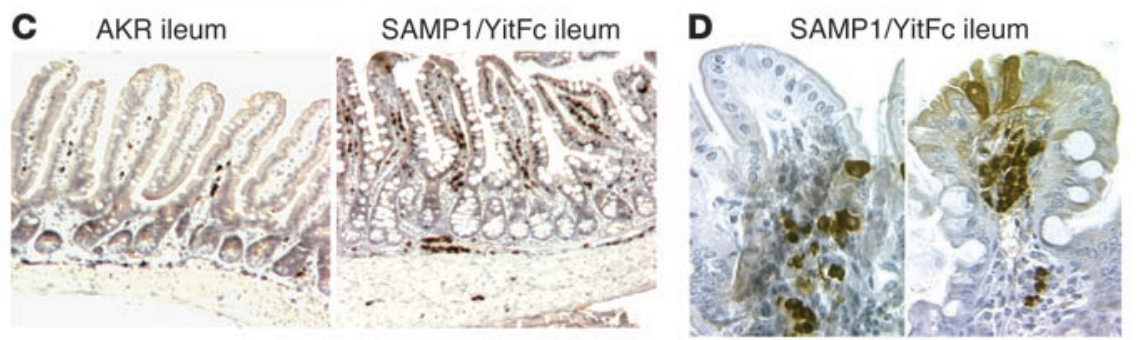

\section{Figure 6}

SAMP1/YitFc MLN and ileum contain large populations of IgA ${ }^{+}$cells. (A) SAMP1/YitFc MLN (top) contain considerably more IgA-secreting cells (dark brown spots) and soluble IgA (diffuse light brown) than do AKR MLN (bottom), as shown by immunostaining of paraffin-embedded MLN sections. (B) Left, representative dot plots of B220 versus IgA expression, with quadrant percentages (mean \pm SEM), demonstrating an increase in mature $B$ cells (B220 hi) and plasmablasts (B220 int) expressing IgA in SAMP1/YitFc MLNs $(n=13)$ versus AKR MLNs $(n=6)$. *Significantly greater $(P<0.05)$ than AKR cell percentage. Right, IgM+'-gated dot plots, with quadrant percentages (mean $\pm \mathrm{SEM}$ ), showing that less than $20 \%$ of SAMP $1 /$ YitFc $(n=13)$ or AKR $(n=6)$ MLN $B$ cells have a CD23-lgD- B1 cell phenotype. (C) Low-power view (magnification, $\times 10$ ) of paraffin-embedded ileal sections immunostained for $\lg \mathrm{A}$, showing increased concentrations of $\lg \mathrm{A}$ secreting cells (dark brown spots) throughout and increased soluble IgA within the villi (diffuse light brown) in SAMP1/YitFc versus AKR ilea. (D) High-power views (magnification, $\times 40$ ) show IgAsecreting cells and soluble IgA focally concentrated in the base (left) and the tip (right) of two villi within SAMP1/YitFc ilea. of $\mathrm{T}_{\text {reg }}$ cells that prevent inflammation in adoptively transferred colitis models $(15,36,37)$, but they cannot prevent ileitis in our adoptive transfer model. The lack of anti-inflammatory capacity of SAMP1/YitFc $\mathrm{T}_{\text {reg }}$ cells in the adoptive transfer model suggests a failure of regulatory pathways in the donor SAMP1/YitFc ileitis model as well.

Wild-type AKR MLN CD4 ${ }^{+} \mathrm{T}$ cells, presumably containing functional $\mathrm{T}_{\text {reg }}$ populations, did not prevent ileitis when cotransferred into SCID mice with SAMP1/YitFc CD4 ${ }^{+} \mathrm{T}$ cells. SAMP1/YitFc $\mathrm{T}_{\text {reg }}$ cells $\left(\mathrm{CD} 45 \mathrm{RB}^{10} \mathrm{CD} 25^{+}\right)$prevented colitis development. Furthermore, SAMP1/YitFc $\alpha_{\mathrm{E}}^{+} \mathrm{CD} 4^{+} \mathrm{T}$ cells suppressed $\alpha_{\mathrm{E}}{ }^{-} \mathrm{CD} 4^{+} \mathrm{T}$ cell proliferation in vitro. The $\alpha_{\mathrm{E}}^{+} \mathrm{CD} 4^{+}$and $\mathrm{CD} 25^{+} \mathrm{CD} 4^{+} \mathrm{T}$ cells represent overlapping subsets of $\mathrm{T}_{\text {reg }}$ cells, and the ability of SAMP1/ YitFc $\alpha_{\mathrm{E}}{ }^{+} \mathrm{CD} 4^{+} \mathrm{T}$ cells to block colitis was not directly tested. Given that SAMP1/YitFc $\alpha_{\mathrm{E}}{ }^{+} \mathrm{CD} 4^{+} \mathrm{T}$ cells block effector $\mathrm{T}$ cell proliferation, and that previous studies have shown that $\alpha_{\mathrm{E}}{ }^{+} \mathrm{CD} 25^{+} \mathrm{T}_{\text {reg }}$ cells are more effective at preventing colitis than is the $\alpha_{\mathrm{E}}{ }^{-} \mathrm{CD} 25^{+}$subset (15), it is likely that SAMP1/YitFc $\alpha_{\mathrm{E}}{ }^{+}$cells block colitis at least as well as SAMP1/YitFc CD25 $5^{+}$cells. Taken together, these findings strongly suggest that aberrant proinflammatory signals that

\section{Figure 7}

B cell population expansion correlates with disease severity in SAMP1/ YitFc mice. (A) Percentages of CD4+ $T$ cells (black) and CD19+ $B$ cells (white) within MLNs (mean \pm SEM) of individual SAMP1/YitFc mice $(n=20)$ in which the total inflammatory score of the distal ileum was greater than 10 (mean $\pm \mathrm{SEM}=15 \pm 1 ; n=14$ ) or less than 10 (mean $\pm \mathrm{SEM}=5 \pm 1 ; n=6)$. ${ }^{*} \mathrm{CD} 4{ }^{+} \mathrm{T}$ cell and $\mathrm{B}$ cell percentages are significantly different $(P<0.02)$ between the two groups. (B) The percentage of B cells in MLNs of mice $(n=20)$ correlates $(r=0.7)$ with the villus distortion component of the histopathological scoring index. override anti-inflammatory pathways, and not inherently defective regulatory cells, are likely the cause of SAMP1/YitFc ileitis.

The increased B cells present within the MLNs of SAMP1/YitFc mice expressed GITRL, increased the severity of adoptively transferred ileitis, and abrogated $\mathrm{T}_{\text {reg }}$ cell function in vitro. Therefore, $\mathrm{B}$ cells may be the primary cell population responsible for overriding anti-inflammatory or regulatory signals in vivo and promoting the development of SAMP1/YitFc ileitis. Alternatively, or in addition, as homing of $\mathrm{T}$ cells to the ileum versus the colon requires differential chemokine receptor expression (38), colitis-preventing $\mathrm{T}_{\mathrm{reg}}$

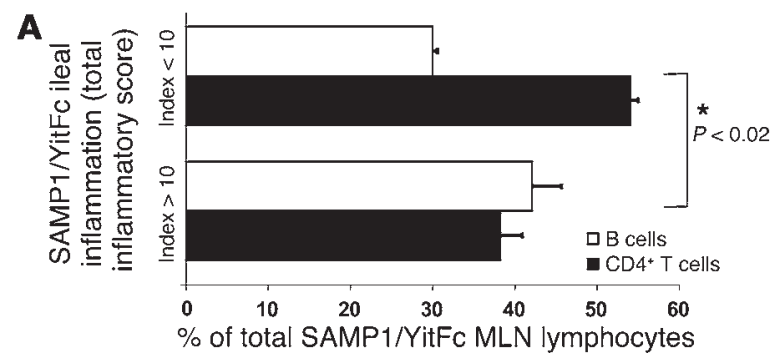

B

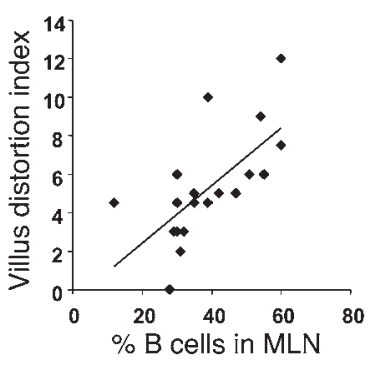


A

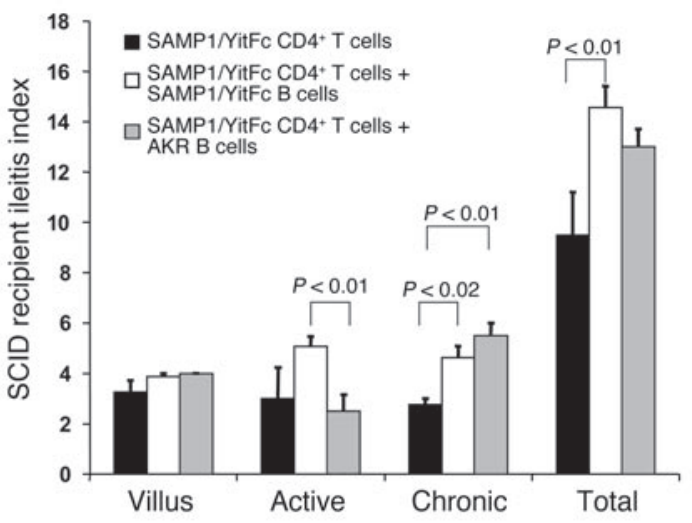

B

B $\quad$ GR-1 (PMN)

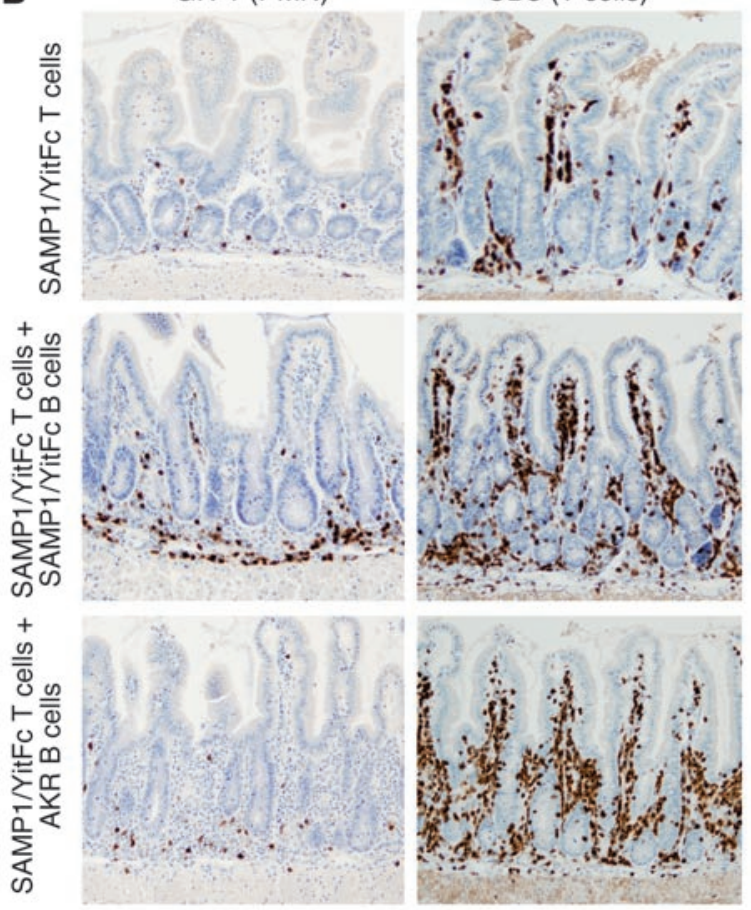

cells may not be effective in curing SAMP1/YitFc ileitis due to an inability to control leukocyte trafficking to or activation within the ileum. The finding that SAMP1/YitFc $\alpha_{\mathrm{E}}{ }^{+} \mathrm{CD} 4^{+} \mathrm{T}$ cells are incapable of decreasing the severity of ileitis adoptively transferred by effector $T$ cell populations even in the absence of $B$ cells suggests that $T_{\text {reg }}$ cells may not be capable of regulating ileal inflammation. However, other populations of APCs native to SCID ileum may also express GITRL (22) and convey a partial ileal-specific inhibition of $T_{\text {reg }}$ cell function that is further enhanced by transfer of SAMP1/YitFc MLN B cells.

The positive correlation between $\alpha_{\mathrm{E}}^{+} \mathrm{CD} 4^{+} \mathrm{T}$ cell and B cell numbers in SAMP1/YitFc MLNs suggests that $\alpha_{\mathrm{E}}{ }^{+} \mathrm{CD} 4^{+} \mathrm{T}$ cells may act as $T_{\mathrm{FH}}$ cells in vivo to produce $\mathrm{B}$ cell population expansion and IgA production. The high level of ICOS expression on $\alpha_{\mathrm{E}}{ }^{+} \mathrm{CD} 4^{+} \mathrm{T}$ cells supports this hypothesis. Alternatively, this correlation and the seemingly paradoxical increase in $\alpha_{\mathrm{E}}{ }^{+} \mathrm{CD} 4^{+}$regulatory cells in diseased versus normal mice could be explained by a model in which $\mathrm{B}$ cell population expansion precedes and induces the expansion of $\alpha_{\mathrm{E}}^{+} \mathrm{CD} 4^{+} \mathrm{T}$ cell populations through GITR/GITRL-mediated interactions, which have been shown previously to induce IL-2 respon-

\section{Figure 8}

Cotransfer of MLN B cells increases ileitis severity in the CD4+ $\mathrm{T}$ cell adoptive transfer model. (A and B) A total of $5 \times 10^{5} \mathrm{CD} 4^{+} \mathrm{T}$ cells from pooled SAMP1/YitFc MLNs $(n=6)$ were injected intraperitoneally into SCID mice either alone $(n=4)$ or in combination with $2 \times 10^{6}$ SAMP1/YitFc $(n=8)$ or AKR $(n=4)$ MLN B cells intravenously. (A) After 6 weeks, SCID mice receiving both SAMP1/YitFc T cells and SAMP1/YitFc B cells had higher chronic and total inflammatory scores than those of mice receiving $T$ cells alone, while mice receiving $A K R$ $B$ cells and SAMP1/YitFc T cells had an intermediate phenotype. (B) Immunostaining of paraffin-embedded ileal sections, revealing an increase in $\mathrm{T}$ cell (CD3+ cells) infiltrates in SCID mice receiving cotransfer of $B$ cells from either strain compared with that of mice receiving $T$ cells alone, and an increased neutrophil (GR-1+ cells) infiltrate in mice receiving specifically SAMP1/YitFc B cells. PMN, polymorphonuclear granulocytes.

siveness and proliferation in $\mathrm{T}_{\text {reg }}$ cells. These two possibilities may not be mutually exclusive, as interactions between T cells and B cells are known to elicit downstream effects in both cell types (39).

SAMP1/YitFc MLN B cells may also contribute to ileal inflammation through the production of immunoglobulin that recognizes specific antigenic epitopes. Neutrophil infiltration within the ileal submucosa was increased in SCID mice receiving SAMP1/YitFc T cells and SAMP1/YitFc B cells compared to the level of infiltration in mice receiving SAMP1/YitFc T cells and AKR B cells. This increased infiltration could result from the formation of specific immune complexes, which contribute to adhesion and tissue recruitment of neutrophils through a Mac-1 integrin-dependent pathway (40).

The spontaneous colitis seen in $\mathrm{C} 3 \mathrm{H} / \mathrm{HeJBir}$ mice is associated with a mainly IgG2a response to enteric bacteria (8). However, the inflammation in these mice is limited to the cecum and the colon (41), and thus immunoglobulin specificity may be different from that of Crohn-like ileitis. While some studies indicate that mucosal IgG, not IgA, is elevated in Crohn patients (7), other studies suggest that serum IgA, but not IgG, immunoreactivity is stronger in Crohn patients than in controls (42). Relative to normal individuals, many Crohn patients have elevated serum IgA specifically recognizing HupB, a mycobacterial homolog of the antigen recognized by antineutrophil cytoplasmic antibody (pANCA) (43); I2, a bacterial transcription factor (44); and Saccharomyces cerevisiae subspecies (45).

Under homeostatic conditions, IgA is translocated across intestinal epithelial cells to the luminal surface, where it prevents bacteria from exiting the lumen, thereby functioning to prevent initiation of immune responses (9). From this perspective, it makes sense that $\mathrm{T}_{\text {reg }}$ cells, which produce IgA-promoting TGF- $\beta$ in other models, would drive IgA production as part of their anti-inflammatory repertoire (46). However, most IgA that binds to enteric bacteria flora is derived from B1 cells, whereas B2-derived IgA like that present in SAMP1/YitFc MLNs recognizes potentially pathogenic, invasive bacteria and aids in their elimination $(47,48)$. In SAMP1/YitFc ileitis, where epithelial barrier integrity is likely diminished as part of the ongoing inflammatory process, the coating of invasive bacteria by IgA may initiate proinflammatory responses through opsonization or immune complex formation, as discussed above. This role, if relevant, may also be played by antibodies of other isotypes, such as IgM or IgG1, which are also increased in SAMP1/YitFc MLNs.

$\mathrm{B}$ cells themselves have recently been shown to directly modulate intestinal inflammation. Backcrossing $T C R^{-/-}$mice with $I g \mu^{-/-}$mice results in mice with more severe chronic colitis than 
that seen in mice that are $T C R^{-/-}$alone, and reconstitution of $T C R^{-/-} I g \mu^{-/-}$mice with mature B cells ameliorates this increased inflammation severity (26). The anti-inflammatory B cell subset in this model expresses IL-10 and high levels of CD1D, a marker of B1 cell-like spleen marginal zone cells $(6,49)$. B cells involved in colitis are likely quite different than $B$ cells that participate in ileitis, as trafficking of B cell lineage subsets to colon versus ileum also relies on distinct pathways $(50,51)$. CD45RB hi $\mathrm{T}$ cells from If $n-\gamma^{-/-}$mice can induce ileitis when transferred into SCID mice only when cotransferred with B cells (52), consistent with our data suggesting that the proinflammatory activity of intestinal B cells may be particularly relevant in the ileum. Furthermore, in the Gai2 $2^{-/}$model of IBD, a selective deficit in B1 cells and an increase in $\mathrm{B} 2$ cells are linked to the development of intestinal inflammation (5). The present findings are consistent with a model in which mucosal B2 cells are proinflammatory and B1 cells are anti-inflammatory in the context of IBD.

In conclusion, we have established a link between an increased regulatory $\mathrm{CD}^{+} \mathrm{T}$ cell subset expressing the $\alpha_{\mathrm{E}} \beta_{7}$ integrin, an expanded MLN B cell population, and elevated serum IgA in SAMP1/YitFc mice. The increased number of IgA-expressing MLN B cells correlates with severity of inflammation in SAMP1/YitFc host ileitis. SAMP1/YitFc $\mathrm{T}_{\text {reg }}$ cells are incapable of modulating the severity of adoptively transferred ileitis, but can prevent colitis. SAMP1/YitFc MLN B cells cotransferred with $\mathrm{CD}^{+} \mathrm{T}$ cells increase the severity of overall ileitis, and specifically $\mathrm{T}$ cell infiltration, in SCID mice. These B cells express GITRL and abrogate $\alpha_{\mathrm{E}}{ }^{+} \mathrm{CD} 4^{+} \mathrm{T}$ cell regulatory function in vitro. Taken together, our results demonstrate the importance of $\mathrm{T}$ cell/B cell interactions and $\mathrm{B}$ cell function in the pathogenesis of a Crohn-like murine ileitis model.

\section{Methods}

Mice. SAMP1/YitFc mice, a substrain of the SAMP1/Yit line (27), were obtained from established colonies at the University of Virginia Health Science Center vivarium (Charlottesville, Virginia, USA). Age-matched wild-type AKR/J mice and 6- to 8-week-old SCID mice on the $\mathrm{C} 3 \mathrm{H} / \mathrm{HeJ}$ background were obtained from Jackson Laboratories (Bar Harbor, Maine,
USA). All animals were housed in a specific pathogen-free facility, and all experiments were approved by the institutional committee for animal use.

Flow cytometry. MLNs were crushed through $70-\mu \mathrm{m}$ filters into staining buffer consisting of PBS with $2 \%$ FCS. Cells were counted using Trypan Blue (Sigma-Aldrich, St. Louis, Missouri, USA). Cells were stained with combinations of biotinylated anti-mouse GITR (BAF524) (R\&D Systems, Minneapolis, Minnesota, USA); streptavidin-allophycocyanin; FITC-, phycoerythrin- (PE-), peridinine chlorophyll protein-, allophycocyanin-, or biotin-labeled rat anti-mouse CD4 (RM4-5), CD8 (53-6.7), CD19 (1D3), $\alpha \mathrm{E}$ (M290), $\beta_{7}$ (M293), ICOS (7E.17G9), CD25 (PC61), L-selectin (MEL-14), CD45RB (16a), B220 (RA3-6B2), IgM (R6-60.2), IgA (C10-3), IgD (11-26c.2a), and CD23 (B3B4); and hamster anti-mouse CD69 (H1.2F3) (BD Biosciences - Pharmingen, San Diego, California, USA). Cells were stained for 20-30 minutes at $4{ }^{\circ} \mathrm{C}$, washed twice in staining buffer and fixed in $1 \%$ paraformaldehyde. Flow cytometry data were acquired on a FACSCalibur flow cytometer with CellQuest software (BD Biosciences, San Diego, California, USA) and were analyzed with WinMIDI 2.8 (J. Trotter, Scripps Research Institute, La Jolla, California, USA).

Cell isolations and adoptive transfer. SAMP1/YitFc or AKR MLN CD4 $4^{+} \mathrm{T}$ cells were isolated magnetically by positive selection with anti-mouse $\mathrm{CD} 4$ microbeads or by negative selection with the mouse $\mathrm{CD}^{+}$negative isolation kit (Miltenyi Biotec, Auburn, California, USA). All selections were performed according to the manufacturer's instructions. B cells were isolated by positive selection with anti-mouse CD19 microbeads or by negative selection with anti-mouse CD43 microbeads (Miltenyi Biotec). By flow cytometry, B cell and CD4+ $\mathrm{T}$ cell fractions were more than $97 \%$ and $95 \%$ pure, respectively. The $\alpha_{\mathrm{E}}{ }^{+}$and $\alpha_{\mathrm{E}}^{-}$subsets were isolated either before or after $\mathrm{CD}^{+}{ }^{+}$cell selection with FITC- or PE-labeled rat anti-mouse $\alpha_{\mathrm{E}}$ and either the anti-FITC multisort kit or anti-PE microbeads (Miltenyi Biotec). By flow cytometry, the $\alpha_{E}{ }^{-}$and $\alpha_{E}{ }^{+}$populations were typically more than $97 \%$ and $80 \%$ pure, respectively. $\mathrm{CD} 25^{+} \mathrm{CD} 4^{+}$and $\mathrm{CD} 25^{-} \mathrm{CD} 4^{+} \mathrm{T}$ cells were separated with PE-anti-mouse CD25 and anti-PE microbeads, whereas $\mathrm{CD} 45 \mathrm{RB}^{\text {hi }} \mathrm{CD}^{+}$and $\mathrm{CD} 45 \mathrm{RB}^{\text {lo }} \mathrm{CD} 4^{+} \mathrm{T}$ cells were separated using labeling with FITC-anti-mouse CD45RB and FACS sorting with the FACSVantage system (BD Biosciences - Pharmingen).

For adoptive transfer, cells were counted, washed, and resuspended in PBS for injection into SCID recipients. T cells and B cells were
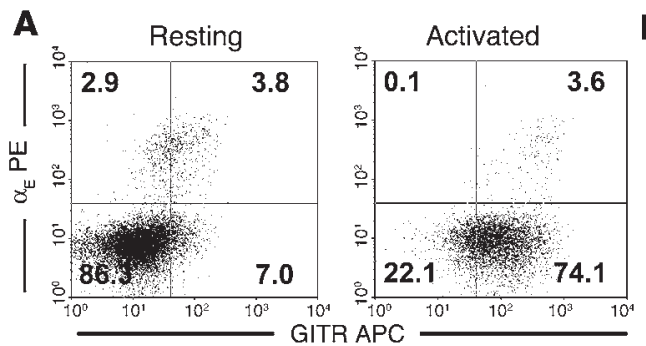

C

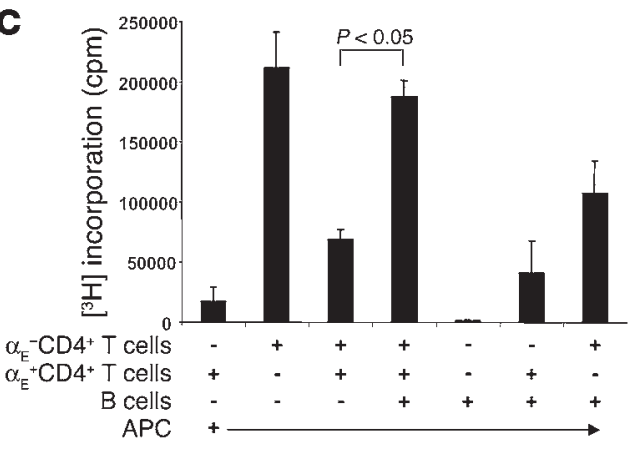

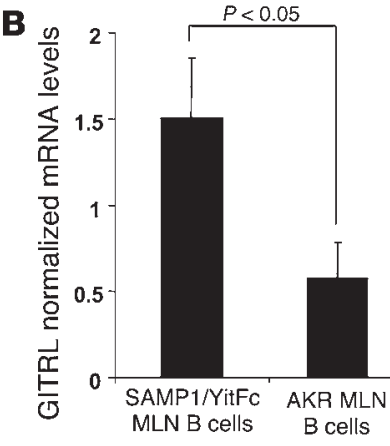

\section{Figure 9}

SAMP1/YitFc MLN B cells block $T_{\text {reg }}$ function in vitro. (A) Increased expression of GITR on freshly isolated or 24 hour-activated (anti-CD3) $\alpha_{E}{ }^{+} C D 4^{+}$versus $\alpha_{E}{ }^{-C D} 4^{+} T$ cells. (B) Increased mRNA expression of GITRL, measured by real-time RT-PCR and normalized to total $18 \mathrm{~S}$ rRNA, on isolated MLN B cells from SAMP1/YitFc mice $(n=8)$ versus AKR mice $(n=6)$. (C) B cells expressing GITRL block $\alpha_{E}{ }^{+} T_{\text {reg }}$ cell function. SAMP1/YitFc irradiated splenic APCs $\left(1 \times 10^{5}\right.$ cells/well $)$ were cultured with combinations of MLN $\alpha_{E}{ }^{+} C D 4^{+} T$ cells, $\alpha_{E}{ }^{-} C D 4^{+} T$ cells, and $B$ cells $\left(5 \times 10^{4}\right.$ each) stimulated for 3 days with immobilized anti-CD3. $\left[{ }^{3} \mathrm{H}\right]$ thymidine was added to the cultures 24 hours before analysis. The $\alpha_{E^{+}} T_{\text {reg }}$ cells blocked the proliferation of effector T cells, while the addition of $B$ cells to the coculture blocked the $T_{\text {reg }}$ cell-mediated inhibition. Data reflect mean \pm SEM of two independent experiments. 
injected in $500 \mu \mathrm{l}$ of PBS at doses of $5 \times 10^{5}$ cells intraperitoneally and $2 \times 10^{6}$ cells i.v. (tail vein), respectively. The ilea and/or colons of SCID recipients were harvested 6 weeks after transfer.

Cell culture, cytokine analysis, and $T$ cell proliferation assays. Cells were cultured in 96-well plates at $37^{\circ} \mathrm{C}$ with $5 \% \mathrm{CO}_{2}$ in RPMI media containing $10 \% \mathrm{FCS}$, $100 \mathrm{U} / \mathrm{ml}$ penicillin, $100 \mu \mathrm{g} / \mathrm{ml}$ streptomycin, and $10 \mu \mathrm{g} / \mathrm{ml}$ plate-bound anti-mouse CD3 (145-2C11) (BD Biosciences - Pharmingen) with or without $2 \mu \mathrm{g} / \mathrm{ml}$ anti-mouse CD28 (37.51) (BD Biosciences - Pharmingen). For cytokine analysis, $\mathrm{T}$ cell subsets were stimulated with plate-bound antiCD3 and soluble anti-CD28 and were cultured for 48 hours at a density of $10^{5}$ cells/well in $100 \mu \mathrm{l}$ of media. Supernatants were assayed for TNF- $\alpha$, IL-2, and IL-10 levels by cytometric bead array using the mouse inflammation and mouse Th1/Th2 cytokine kits (BD Biosciences - Pharmingen) according to the manufacturer's instructions. In T cell/B cell coculture experiments, $10^{5}$ MLN CD4 ${ }^{+}$T cells and $10^{5}$ MLN B cells were cultured in $200 \mu \mathrm{l} /$ well for 3,7 , or 11 days in wells containing plate-bound anti-CD3 with or without soluble anti-CD28.

For proliferation assays, $10^{5}$ irradiated (3,000 rad) splenic APCs were cultured for 3 days with plate-bound anti-CD3 $(10 \mu \mathrm{g} / \mathrm{ml})$ and combinations of $\alpha_{\mathrm{E}}{ }^{+} \mathrm{CD} 4^{+} \mathrm{T}$ cells, $\alpha_{\mathrm{E}}{ }^{-} \mathrm{CD} 4^{+} \mathrm{T}$ cells, and B cells, each at a density of $5 \times 10^{4}$ cells/well. Each condition was assayed in triplicate. Incorporation of $\left[{ }^{3} \mathrm{H}\right]$ thymidine $(1 \mu \mathrm{Ci} /$ well) (MP Biomedicals, Irvine, California, USA) during the last 24 hours of culture was measured with a Harvester 96 (Tomtec, Humden, Connecticut, USA) for cell harvest and a 1450 Microbeta Scintillation Counter (PerkinElmer, Gaithersburg, Maryland, USA).

Measurement of immunoglobulin levels. The SBA Clonotyping System ELISA (Southern Biotech, Birmingham, Alabama, USA) was used according to the manufacturer's instructions for measurement of immunoglobulin isotypes in $0.5 \mu \mathrm{l}$ of serum samples and $20-40 \mu \mathrm{l}$ of coculture supernatants diluted to a final volume of $100 \mu \mathrm{l}$ in PBS containing $1 \%$ bovine serum albumin. The concentration of goat anti-mouse Ig capture antibody used was $10 \mu \mathrm{g} / \mathrm{ml}$, while horseradish peroxidase-labeled goat anti-mouse IgA detection antibody was used at a dilution of 1:1,000. Plates were developed for 10-20 minutes with 2,2'-azino-bis 3-ethylbenzthiazoline-6-sulfonic acid substrate, and optical density readings were obtained at $405 \mathrm{~nm}$ on a microplate reader (Labsystems, Needham Heights, Massachusetts, USA). Samples were measured in triplicate. MLN immunoglobulin concentrations were determined with the mouse immunoglobulin isotyping cytometric bead array (BD Biosciences - Pharmingen). MLN contents were strained into $5 \mathrm{ml}$ of master buffer and were centrifuged for $5 \mathrm{~min}(350 \mathrm{~g})$. Supernatants were removed and assayed according to the manufacturer's instructions, using 1:100 and 1:1 dilutions of supernatant for detection of $\kappa$ and $\lambda$ light chain immunoglobulins, respectively.

Histology and immunohistochemistry. For histology, the ileum was flushed, opened, rolled longitudinally, and fixed in Bouin's fixative (Fisher, Newark, Delaware, USA). MLNs were fixed directly in Bouin's fixative. All tissues were embedded in paraffin and were cut into sections 3-5 $\mu \mathrm{m}$ in thickness. Hemotoxylin- and eosin-stained ileal sections were evaluated by a trained histopathologist "blinded" to sample identity, using a standardized histological scoring system as described (33). For immunohistochemistry (53), sections were deparaffinized, blocked for 1 hour (PBS with avidin, $10 \%$ normal goat serum and $0.5 \%$ gelatin), washed, and incubated over- night at $4^{\circ} \mathrm{C}$ with $1 \mu \mathrm{g} / \mathrm{ml}$ of biotinylated goat anti-mouse IgA (Southern Biotech), $0.2 \mu \mathrm{g} / \mathrm{ml}$ of purified rat anti-mouse GR-1 (RB6-8C5) (American Type Culture Collection, Manassas, Virginia, USA), or $0.7 \mu \mathrm{g} / \mathrm{ml}$ of purified goat polyclonal anti-mouse CD3 (M20) (Santa Cruz Biotechnology, Santa Cruz, California, USA). For GR1 and CD3 stains, sections were subsequently washed and incubated with $5 \mu \mathrm{g} / \mathrm{ml}$ biotinylated rabbit anti-rat and biotinylated rabbit anti-goat secondary antibodies, respectively (Vector Laboratories, Burlingame, California, USA). Sections were then incubated with avidin-biotin peroxidase complexes, developed with 3,3'-diaminobenzidine substrate (Vector Laboratories) and counterstained with hematoxylin. Images were acquired with an Olympus BH-2 microscope (Melville, New York, USA) and Image-Pro Plus (Media Cybernetics, Carlsbad, California, USA) software.

Real-time RT-PCR. B cell RNA was extracted using RNAeasy (Qiagen, Valencia, California, USA), reverse-transcribed with the Superscript kit (Invitrogen, California, USA), and assayed for GITRL expression by probebased real-time PCR with a Smart Cycler (Cepheid, Sunnyvale, California, USA). The primer sequences were 5'-CCCAAGGTGTCCAGAATGAAG-3' and 5'-AGTCAGCATGGTTGAGTGAGATG-3'. The probe sequence was 5'TET-CTGGAGCAAGAAGATCCAGGATACTG-3' (GITRL probe) (Integrated DNA Technologies, Coralville, Iowa, USA). The PCR cycling conditions were $95^{\circ} \mathrm{C}$ ( 300 seconds) followed by 40 cycles of $95^{\circ} \mathrm{C}$ ( 15 seconds $), 60^{\circ} \mathrm{C}$ (30 seconds), and $72^{\circ} \mathrm{C}$ (30 seconds). Critical threshold (CT) values and standard curves were used to estimate starting mRNA levels. Values were normalized against $18 \mathrm{~S}$ rRNA CT values generated with the preoptimized $18 \mathrm{~S}$ rRNA primers and probe set (Applied Biosystems).

Statistics. Statistical analysis was performed with the two-tailed unpaired Student's $t$ test, the Mann-Whitney rank sum test, or one-way ANOVA for multiple comparisons with the Student-Newman-Keuls method. Statistical analysis for real-time RT-PCR data was performed with the one-tailed unpaired Student's $t$ test. Statistical significance was set at $P$ less than 0.05 . Coefficients of determination, $R^{2}$, were determined using linear regression.

\section{Acknowledgments}

The authors would like to acknowledge Leslie Hancock for animal husbandry; Sharon Hoang and Kirstin Skaar for histology; Christopher Moskaluk and James Mize for inflammation scoring; John Sanders, Matthew Stark, R. Cartland Burns, Jennifer Mounts, Alda Vidrich, Angela Best, and Anthony Bruce for technical assistance; and Satoshi Matsumoto for providing the original SAMP1/Yit breeding pairs. Supported by a Crohn's and Colitis Foundation of America grant to M. Naganuma; and NIH grants P01 DK 57880 to F. Cominelli, K26 RR 00175 to P.B. Ernst, the Immunology and Cell Isolation Core of P30 DK 56703, and GM07267-23 to the University of Virginia and T.S. Olson.

Received for publication December 17, 2003, and accepted in revised form June 22, 2004.

Address correspondence to: Klaus Ley, Health Science Center Box 801394, Charlottesville, Virginia 22908, USA. Phone: (434) $243-$ 9966; Fax: (434) 924-2828; E-mail: klausley@virginia.edu.
1. Elson, C.O. 2002. Genes, microbes, and T cells-new therapeutic targets in Crohn's disease. N. Engl. J. Med. 346:614-616.

2. Bouma, G., and Strober, W. 2003. The immunological and genetic basis of inflammatory bowel disease. Nat. Rev. Immunol. 3:521-533.

3. Dieckgraefe, B.K., and Korzenik, J.R. 2002. Treatment of active Crohn's disease with recombinant human granulocyte-macrophage colony-stimulat- ing factor. Lancet. 360:1478-1480.

4. Boone, D.L., and Ma, A. 2003. Connecting the dots from Toll-like receptors to innate immune cells and inflammatory bowel disease. J. Clin. Invest. 111:1284-1286. doi:10.1172/JCI200318545.

5. Dalwadi, H., et al. 2003. B cell developmental requirement for the $\mathrm{G}$ alpha $\mathrm{i} 2$ gene. J. Immunol. 170:1707-1715.

6. Mizoguchi, A., Mizoguchi, E., Takedatsu, H., Blum- berg, R.S., and Bhan, A.K. 2002. Chronic intestinal inflammatory condition generates IL-10-producing regulatory $B$ cell subset characterized by CD1d upregulation. Immunity. 16:219-230.

7. Macpherson, A., Khoo, U.Y., Forgacs, I., PhilpottHoward, J., and Bjarnason, I. 1996. Mucosal antibodies in inflammatory bowel disease are directed against intestinal bacteria. Gut. 38:365-375.

8. Brandwein, S.L., et al. 1997. Spontaneously colitic 
$\mathrm{C} 3 \mathrm{H} / \mathrm{HeJBir}$ mice demonstrate selective antibody reactivity to antigens of the enteric bacterial flora. J. Immunol. 159:44-52.

9. Macpherson, A.J., Hunziker, L., McCoy, K., and Lamarre, A. 2001. IgA responses in the intestinal mucosa against pathogenic and non-pathogenic microorganisms. Microbes Infect. 3:1021-1035.

10. Kroese, F.G., et al. 1989. Many of the IgA producing plasma cells in murine gut are derived from selfreplenishing precursors in the peritoneal cavity. Int. Immunol. 1:75-84.

11. Berland, R., and Wortis, H.H. 2002. Origins and functions of B-1 cells with notes on the role of CD5. Annu. Rev. Immunol. 20:253-300.

12. Breitfeld, D., et al. 2000. Follicular B helper T cells express CXC chemokine receptor 5, localize to B cell follicles, and support immunoglobulin production. J. Exp. Med. 192:1545-1552.

13. McAdam, A.J., et al. 2001. ICOS is critical for CD40-mediated antibody class switching. Nature. 409:102-105.

14. Singh, B., et al. 2001. Control of intestinal inflammation by regulatory $\mathrm{T}$ cells. Immunol. Rev. 182:190-200

15. Lehmann, J., et al. 2002. Expression of the integrin alpha Ebeta 7 identifies unique subsets of CD25+ as well as CD25- regulatory T cells. Proc. Natl. Acad. Sci. U. S. A. 99:13031-13036.

16. Cepek, K.L., et al. 1994. Adhesion between epithelial cells and T lymphocytes mediated by E-cadherin and the alpha E beta 7 integrin. Nature. 372:190-193.

17. Strauch, U.G., et al. 2001. Integrin alpha $\mathrm{E}(\mathrm{CD} 103)$ beta 7 mediates adhesion to intestinal microvascular endothelial cell lines via an E-cadherin-independent interaction. J. Immunol. 166:3506-3514.

18. Schon, M.P., et al. 1999. Mucosal T lymphocyte numbers are selectively reduced in integrin alpha $\mathrm{E}$ (CD103)-deficient mice. J. Immunol. 162:6641-6649.

19. McHugh, R.S., et al. 2002. CD4(+)CD25(+) immunoregulatory $\mathrm{T}$ cells: gene expression analysis reveals a functional role for the glucocorticoidinduced TNF receptor. Immunity. 16:311-323.

20. Kwon, B., Kim, B.S., Cho, H.R., Park, J.E., and Kwon, B.S. 2003. Involvement of tumor necrosis factor receptor superfamily(TNFRSF) members in the pathogenesis of inflammatory diseases. Exp. Mol. Med. 35:8-16.

21. Kim, J.D., et al. 2003. Cloning and characterization of GITR ligand. Genes Immun. 4:564-569.

22. Tone, M., et al. 2003. Mouse glucocorticoidinduced tumor necrosis factor receptor ligand is costimulatory for T cells. Proc. Natl. Acad. Sci. U. S. A. 100:15059-15064.

23. Matsumoto, S., et al. 1998. Inflammatory bowel disease-like enteritis and caecitis in a senescence accelerated mouse P1/Yit strain. Gut. 43:71-78.

24. Bamias, G., et al. 2002. Down-regulation of intes- tinal lymphocyte activation and Th1 cytokine production by antibiotic therapy in a murine model of Crohn's disease. J. Immunol. 169:5308-5314.

25. Kosiewicz, M.M., et al. 2001. Th1-type responses mediate spontaneous ileitis in a novel murine model of Crohn's disease. J. Clin. Invest. 107:695-702.

26. Mizoguchi, E., Mizoguchi, A., Preffer, F.I., and Bhan, A.K. 2000. Regulatory role of mature B cells in a murine model of inflammatory bowel disease. Int. Immunol. 12:597-605.

27. Rivera-Nieves, J., et al. 2003. Emergence of perianal fistulizing disease in the SAMP1/YitFc mouse, a spontaneous model of chronic ileitis. Gastroenterology. 124:972-982.

28. Birkeland, M.L., Johnson, P., Trowbridge, I.S., and Pure, E. 1989. Changes in CD45 isoform expression accompany antigen-induced murine T-cell activation. Proc. Natl. Acad. Sci. U. S. A. 86:6734-6738.

29. Ohgama, J., et al. 1994. Functional studies on MEL14+ and MEL-14- T cells in peripheral lymphoid tissues. Immunobiology. 190:225-242.

30. Lowenthal, J.W., Zubler, R.H., Nabholz, M., and MacDonald, H.R. 1985. Similarities between interleukin-2 receptor number and affinity on activated B and T lymphocytes. Nature. 315:669-672.

31. Mudter, J., Wirtz, S., Galle, P.R., and Neurath, M.F. 2002. A new model of chronic colitis in SCID mice induced by adoptive transfer of CD62L+CD4+ T cells: insights into the regulatory role of interleukin6 on apoptosis. Pathobiology. 70:170-176.

32. Butcher, E.C., Williams, M., Youngman, K., Rott, L. and Briskin, M. 1999. Lymphocyte trafficking and regional immunity. Adv. Immunol. 72:209-253.

33. Burns, R.C., et al. 2001. Antibody blockade of ICAM-1 and VCAM-1 ameliorates inflammation in the SAMP-1/Yit adoptive transfer model of Crohn's disease in mice. Gastroenterology. 121:1428-1436.

34. Tafuri, A., et al. 2001. ICOS is essential for effective T-helper-cell responses. Nature. 409:105-109.

35. Lazarus, N.H., et al. 2003. A common mucosal chemokine (mucosae-associated epithelial chemokine/CCL28) selectively attracts IgA plasmablasts. J. Immunol. 170:3799-3805.

36. Mottet, C., Uhlig, H.H., and Powrie, F. 2003. Cutting Edge: Cure of colitis by CD4(+)CD25(+) regulatory T cells. J. Immunol. 170:3939-3943.

37. Powrie, F., Leach, M.W., Mauze, S., Caddle, L.B., and Coffman, R.L. 1993. Phenotypically distinct subsets of CD4+ T cells induce or protect from chronic intestinal inflammation in C. B-17 scid mice. Int. Immunol. 5:1461-1471.

38. Kunkel, E.J., et al. 2000. Lymphocyte CC chemokine receptor 9 and epithelial thymus-expressed chemokine (TECK) expression distinguish the small intestinal immune compartment: Epithelial expression of tissue-specific chemokines as an organizing principle in regional immunity. J. Exp. Med. 192:761-768.
39. Walker, L.S., Gulbranson-Judge, A., Flynn, S., Brocker, T., and Lane, P.J. 2000. Co-stimulation and selection for T-cell help for germinal centres: the role of CD28 and OX40. Immunol. Today. 21:333-337.

40. Tang, T., et al. 1997. A role for Mac-1 (CDIIb/CD18) in immune complex-stimulated neutrophil function in vivo: Mac-1 deficiency abrogates sustained Fcgamma receptor-dependent neutrophil adhesion and complement-dependent proteinuria in acute glomerulonephritis. J. Exp. Med. 186:1853-1863.

41. Sundberg, J.P., Elson, C.O., Bedigian, H., and Birkenmeier, E.H. 1994. Spontaneous, heritable colitis in a new substrain of $\mathrm{C} 3 \mathrm{H} / \mathrm{HeJ}$ mice. Gastroenterology. 107:1726-1735.

42. Kazemi-Shirazi, L., et al. 2002. IgA autoreactivity: a feature common to inflammatory bowel and connective tissue diseases. Clin. Exp. Immunol. 128:102-109.

43. Cohavy, O., et al. 1999. Identification of a novel mycobacterial histone $\mathrm{H} 1$ homologue $(\mathrm{HupB})$ as an antigenic target of pANCA monoclonal antibody and serum immunoglobulin A from patients with Crohn's disease. Infect. Immun. 67:6510-6517.

44. Sutton, C.L., et al. 2000. Identification of a novel bacterial sequence associated with Crohn's disease. Gastroenterology. 119:23-31.

45. Landers, C.J., et al. 2002. Selected loss of tolerance evidenced by Crohn's disease-associated immune responses to auto- and microbial antigens. Gastroenterology. 123:689-699.

46. Shanahan, F. 2002. Crohn's disease. Lancet. 359:62-69.

47. Kroese, F.G., and Bos, N.A. 1999. Peritoneal B-1 cells switch in vivo to IgA and these IgA antibodies can bind to bacteria of the normal intestinal microflora. Curr. Top. Microbiol. Immunol. 246:343-349; discussion 350

48. Kroese, F.G., de Waard, R., and Bos, N.A. 1996. B-1 cells and their reactivity with the murine intestinal microflora. Semin. Immunol. 8:11-18.

49. Amano, M., et al. 1998. CD1 expression defines subsets of follicular and marginal zone B cells in the spleen: beta 2-microglobulin-dependent and independent forms. J. Immunol. 161:1710-1717.

50. Kunkel, E.J., and Butcher, E.C. 2002. Chemokines and the tissue-specific migration of lymphocytes. Immunity. 16:1-4.

51. Kunkel, E.J., et al. 2003. CCR10 expression is a common feature of circulating and mucosal epithelial tissue IgA Ab-secreting cells. J. Clin. Invest. 111:1001-1010. doi:10.1172/JCI200317244.

52. Dohi, T., et al. 2003. T helper type-2 cells induce ileal villus atrophy, goblet cell metaplasia, and wasting disease in T cell-deficient mice. Gastroenterology. 124:672-682.

53. Bishop, G.G., et al. 2001. Selective $\alpha_{\mathrm{v}} \beta_{3}$-receptor blockade reduces macrophage infiltration and restenosis after balloon angioplasty in the atherosclerotic rabbit. Circulation. 103:1906-1911. 